\title{
Biopolymers in sorbent-based microextraction methods
}

\author{
Idaira Pacheco-Fernández a, b, Diego W. Allgaier-Díaz a, Giulia Mastellone a, c, \\ Cecilia Cagliero ${ }^{\mathrm{c}}$, David Díaz Díaz ${ }^{\mathrm{d}, \mathrm{e}, \mathrm{f}}$, Verónica Pino a, b, * \\ a Departamento de Química, Unidad Departamental de Química Analítica, Universidad de La Laguna (ULL), Tenerife, 38206, Spain \\ ${ }^{\mathrm{b}}$ Instituto Universitario de Enfermedades Tropicales y Salud Pública de Canarias, Universidad de La Laguna (ULL), Tenerife, 38206, Spain \\ ${ }^{\mathrm{c}}$ Dipartamento di Scienza e Tecnologia del Farmaco, Università degli Studi di Torino, Torino, 10125, Italy \\ d Departamento de Química Orgánica, Universidad de La Laguna (ULL), Tenerife, 38206, Spain \\ e Instituto Universitario de Bio-Orgánica Antonio González, Universidad de La Laguna (ULL), Tenerife, 38206, Spain \\ ${ }^{\mathrm{f}}$ Institut für Organische Chemie, Universität Regensburg, Regensburg, 93053, Germany
}

\section{A R T I C L E I N F O}

\section{Article history:}

Available online 10 February 2020

\section{Keywords:}

Biopolymer

Microextraction

Chitosan

Cellulose

Alginate

Agarose

Solid-phase extraction

\begin{abstract}
A B S T R A C T
Since the introduction of the Green Analytical Chemistry guidelines, there has been an increasing concern on the sustainability of sample preparation approaches, particularly if considering they constitute the most time-consuming step of the analytical method and the main source of laboratory wastes. Among the alternatives explored, it is important to highlight the miniaturization of the extraction methods, which has been accompanied by the seek of greener solvents and sorbents. Biopolymers emerge as potential candidates to be used as sorbents in microextraction schemes given their biodegradability, versatility, and easily functionalization. This review offers an overview on biopolymers (chitosan, cellulose, alginate, and agarose) in sorbent-based microextraction approaches, paying attention to the preparation of the sorbent and the format in which biopolymers are incorporated into the sorbent/device, thus implying a specific microextraction approach, their role in the resulting sorbent material, and the reported analytical applications, covering environmental, food, and bioclinical analysis.
\end{abstract} (C) 2020 Elsevier B.V. All rights reserved.

\section{Introduction}

The incorporation of the Green Chemistry principles in the analytical process has emerged as one of the most important research lines within the analytical chemistry community, leading to the development of the green analytical chemistry (GAC) [1]. Current efforts clearly shift to improve the sustainability of the analytical sample preparation stage since it involves numerous tedious steps, it is highly time-consuming, and entails the generation of large amounts of wastes [2]. Among the strategies, (i) the minimization, replacement, or elimination of harmful organic solvents in the analytical sample preparation procedure [3], together with (ii) the improvement of the methodologies by using on-line, automated and microextraction approaches (this way permitting

\footnotetext{
* Corresponding author. Departamento de Química, Unidad Departamental de Química Analítica, Universidad de La Laguna (ULL), Tenerife, 38206, Spain.

E-mail addresses: ipacheco@ull.edu.es (I. Pacheco-Fernández), diego.walter. allgaier.diaz.05@ull.edu.es (D.W. Allgaier-Díaz), giulia.mastellone@edu.unito.it (G. Mastellone), cecilia.cagliero@unito.it (C. Cagliero),ddiazdiaz@ull.edu.es, David. Diaz@chemie.uni-regensburg.de (D.D. Díaz), veropino@ull.edu.es (V. Pino).
}

a decrease of costs, number of steps and analysis time, while allowing the development of high-throughput methods) [4,5], are the most productive approaches followed to comply with the GAC guidelines.

\subsection{Overview of sorbent-based microextraction methods}

Microextraction methods, in which low amounts of both extraction material and sample are used, have been introduced to overcome the sustainability drawbacks of conventional analytical sample preparation techniques [6]. In particular, sorbent-based microextraction approaches have attracted much attention due to the possibility of taking advantage of material science technologies to prepare smart and task-specific solid materials [7,8]. Fig. 1 includes a general scheme of different sorbent-based microextraction strategies, including several representations of most common devices.

Solid-phase extraction (SPE) is a widely used conventional extraction technique, that involves the use of a sorbent material packed in a small column device. A relatively high volume of sample $(0.001-2 \mathrm{~L})$ is passed through the column to ensure 


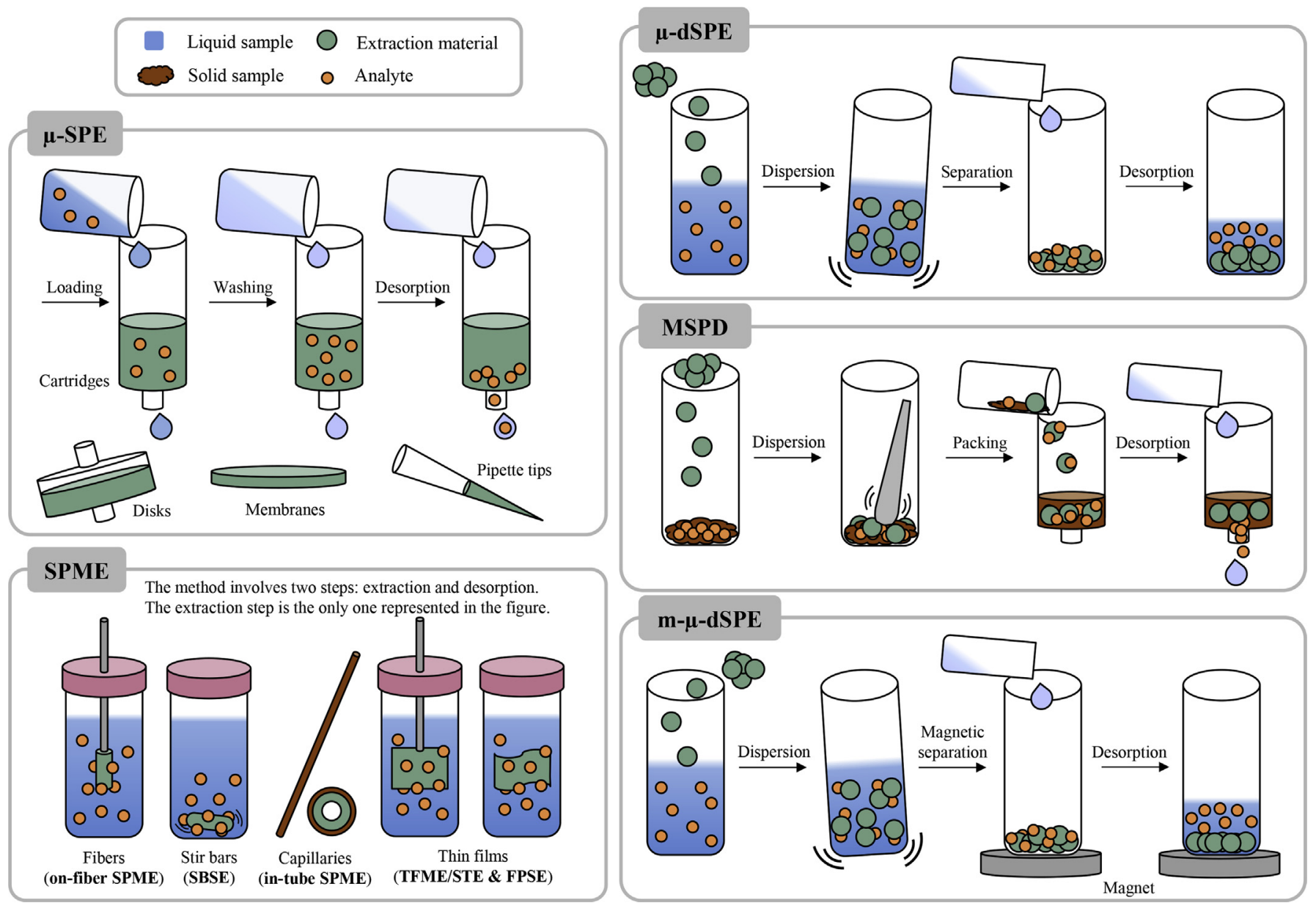

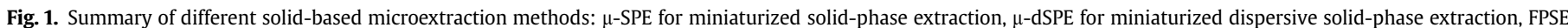

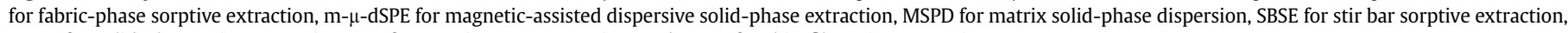
SPME for solid-phase microextraction, STE for sorptive tape extraction, and TFME for thin-film microextraction.

trapping of the analytes by the sorbent. Then, the target compounds are eluted using a low amount of solvent [9]. SPE constitutes the basis for the development of microextraction methods requiring a solid sorbent material.

The miniaturized sorbent-based method most similar to conventional SPE is termed miniaturized SPE ( $\mu$-SPE), in which lower amounts of sorbent $(<500 \mathrm{mg})$ and liquid sample $(<20 \mathrm{~mL})$ are required. Indeed, different configurations apart from cartridges and microcolumns have been proposed with the aim of reducing the amount of sorbent, such as disks, membranes and pipette tips [10].

Despite the benefits of $\mu$-SPE, it requires the activation of the packed sorbent, which involves the consumption of organic solvents, relatively long extraction times due to the slow flow rates required, possible cartridge blocking, etc. In this sense, dispersive $\mu$-SPE ( $\mu$-dSPE) arises as an alternative to overcome these problems [11]. In this case, few $\mathrm{mg}(1-500 \mathrm{mg})$ of the solid sorbent is dispersed in the liquid sample. Then, the sorbent is separated and subjected to desorption using an adequate solvent. This strategy has also been applied for the analysis of solid samples, leading to matrix solid-phase dispersion (MSPD) approach [12]. In this case, both solid sorbent and solid sample are strongly mixed during the microextraction stage, and then packed into a column to perform the desorption step.

However, $\mu$-dSPE and MSPD exhibit some weaknesses, mainly associated to tedious and time-consuming filtration and centrifugation steps. The use of magnetic sorbents in the extraction procedure easily success in dealing with these limitations [13]. In the magnetic-assisted $\mu$-dSPE approach (m- $\mu$-dSPE), the magnetic sorbent is separated from the initial sample or desorption solvent with the aid of an external magnet.

In 1990, Pawliszyn et al. introduced solid-phase microextraction (SPME) as a new non-exhaustive sampling, preconcentration and extraction technique [14]. The original SPME device consisted of a small amount of the active coating material immobilized on the surface of a small fiber, forming a coating of $1 \mathrm{~cm}$ of length with thicknesses up to $100 \mu \mathrm{m}$. In this technique, the fiber is exposed to the sample (or to the headspace) to perform the extraction of the analytes for a prefixed time. Then, the analytes are desorbed from the sorbent either by thermal desorption (in the inlet of a gas chromatograph (GC)) or by using a solvent. SPME offers simplicity, reusability, complete absence of organic solvent in the method if using thermal desorption, automation, and impressive enrichment capacity [5]. However, different SPME designs have been proposed over the years to improve the analytical performance of the original on-fiber configuration [15] and are schematically shown in Fig. 1.

In the in-tube SPME mode, the inner walls of a capillary are coated with a thin layer of the sorbent material but packed and monolith capillaries have also been proposed in recent studies [16]. It was introduced to improve the coupling of the SPME technique with liquid-chromatography (LC) systems. The operating steps in this case resembles that of $\mu$-SPE, following a flow-through procedure. Stir bar sorptive extraction (SBSE) is a technique that can be 
understood as a solution to the small amount of sorbent used in the previous configurations [17]. In this design, a magnetic stir bar is coated with a thicker layer of the sorbent material. This device can be used in both solvent and thermal desorption (with a suitable thermo-desorption unit). Besides an enhancement in the extraction capacity, the use of higher amounts of sorbent in SBSE is also accompanied by long extraction times to reach the equilibrium. Thin-film microextraction (TFME) or sorptive tape extraction (STE), in which the sorbent coats a flat surface or consists of a free membrane with a reduced thickness, was proposed to address this issue $[18,19]$. While high amounts of sorbent are used, the thin film configuration provides a high surface area. In this approach, the membrane is freely dispersed all over the sample or immersed in the sample with the aid of a wire. A variation of the TFME, named fabric-phase sorptive extraction (FPSE), was recently developed and involves the use of a thin film fabric substrate coated with a sorbent prepared using the sol-gel technique [20].

\subsection{Novel materials in sorbent-based microextraction methods}

These advances in sorbent-based microextraction methods have come together with the design and incorporation of new materials able to provide more efficient extractions, while compiling with the requirements of GAC [7,21]. It is important to highlight that methods incorporating these materials must be able to maintain adequate quality analytical performance despite the decrease in the amounts of sorbent and sample. Conventional sorbents in this microextraction strategies are commonly based on polymers, such as polydimethylsiloxane or divinylbenzene, which usually lack of selectivity, exhibit poor extraction capacity towards more polar compounds, and also show poor matrix-compatibility and low thermal stability. In this sense, trends within this research field have been focused on the use of tunable and highly porous materials to prepare smart, advanced and efficient sorbents.

Among the great diversity of sorbents explored, carbonaceous materials, including carbon nanotubes (CNTs), graphene and graphene oxide (GO) have been particularly interesting for the extraction of a wide variety of compounds [7,22]. Magnetic nanoparticles (MNPs) have been fundamental for the development of $\mathrm{m}$ $\mu$-dSPE methods [7,13], while molecularly imprinted polymers (MIPs), aptamers and immunosorbents have been useful for target analysis [22,23]. Sorbent-based microextraction methods have also taken advantage of the synthetic versatility, environmentalsustainability and tunable properties of ionic liquids (ILs) and their derivatives [24]; as well as the high surface area and porosity of metal-organic frameworks (MOFs) $[25,26]$ and covalent organic frameworks (COFs) [27]. Despite the success of all these new materials, there are several concerns regarding their toxicity and/or the harmful effect of the reagents required in their synthetic routes $[3,28,29]$. Therefore, it is imperative to search for truly environmental-friendly alternatives to prepare sorbent-based microextraction sorbents [30].

\subsection{Biopolymers}

Biopolymers are polymers obtained from natural sources [31] and constitute a renewable and biodegradable resource of materials. This group includes polysaccharides, protein and lipids derived from animals and plants, being the polysaccharides the most commonly used due to their water-solubility, gelling, and emulsifying properties. Considering these features, biopolymers may meet both the requirements of GAC and microextraction methods. Indeed, they have been already explored in this field, being the polysaccharides chitosan, cellulose, alginate, and agarose, the most common. Fig. 2 includes a summary on the use of these biopolymers in sorbent-based microextraction methods in the last years, together with the formats/devices in which they are found in these applications. It is important to highlight that, despite their versatility, they have been mainly used in combination with other functional materials as shown in Fig. 2. In fact, there are a reduced number of studies that report the use of the neat biopolymer in the microextraction procedure.

In 2016, Sanagi et al. nicely reviewed the use of agarose and alginate in sample preparation approaches focusing on liquidphase microextraction methods and TFME strategies [32]. Furthermore, the use of cellulose-based sorbents for environmental applications has been recently reviewed by Wu et al. [33]. However, these articles on the topic have been focused on specific microextraction methods or applications, while the use of chitosan has never been covered despite the high number of applications in the recent years. In this sense, this review aims to provide an overview on the state of the art of the incorporation of the four most common biopolymers in sorbent-based microextraction approaches, reviewing the analytical applications reported since 2014 to 2019. Considering the diversity of forms in which these materials has been used, together with the broad variety of composites or hybrid sorbents that have been synthesized, the applications for each biopolymer will be discussed according to the microextraction method developed.

\section{Biopolymers in sorbent-based microextraction schemes}

\subsection{Chitosan}

\subsubsection{Chitosan nature and uses}

Chitin is a natural polysaccharide with $N$-acetyl-D-glucosamine as monomeric unit, that occurs in many living organisms. Chitosan is the deacetylated derivative of chitin, which is obtained under alkaline conditions when the degree of deacetylation is $50 \%$ or higher, leading to the formation of a copolymer with $\mathrm{N}$-acetyl-Dglucosamine and D-glucosamine units, as shown in Fig. 3 [34]. Despite the high abundance of chitin in the nature, its negligible solubility in aqueous media and organic solvents limits its applicability. However, chitosan presents an interesting set of characteristics which makes this pseudo-natural polymer very useful in numerous applications [34,35]. It is soluble in acidic media, leading to the formation of a natural cationic polymer due to the protonation of the amino group, while it is water-insoluble at high $\mathrm{pH}$ values. Besides its non-toxicity and biodegradability, this biopolymer can be easily crosslinked using different reagents and the reactivity of the amino group allows the easy functionalization of its chemical structure. Given the physicochemical features of chitosan, it can be processed as powder, dissolved in solution, while being able to form gels, beads, films or foams

Chitosan has been successfully exploited in cosmetic, pharmaceutical and biomedical applications [35]. Due to its biocompatibility, it has been particularly useful for drug delivery. In this sense, it is not surprising that it has also been explored in analytical methods with extraction purposes. Indeed, it is the most used biopolymer in sorbent-based microextraction schemes in the recent years (Fig. 2).

\subsubsection{Chitosan in sorbent-based microextraction methods}

The use of chitosan will be discussed in terms of its incorporation in the different microextraction methods. In each type, its use as neat material or mainly incorporated in sorbents together with other materials will be detailed, while paying attention to the format of the device.

Several forms of chitosan have been used to prepare a number of packed devices for $\mu$-SPE. Zhu et al. [36] proposed commercial 

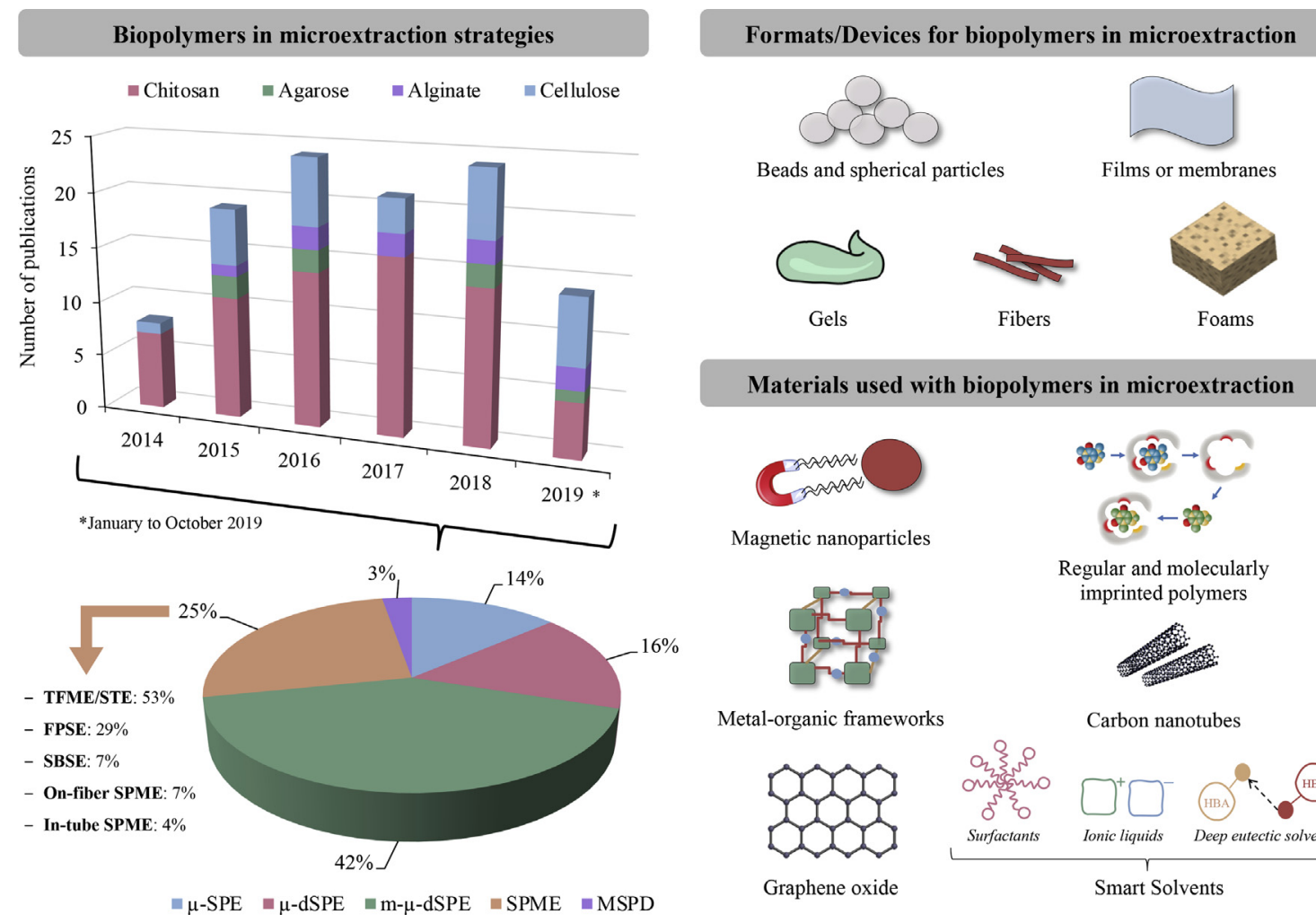

Materials used with biopolymers in microextraction
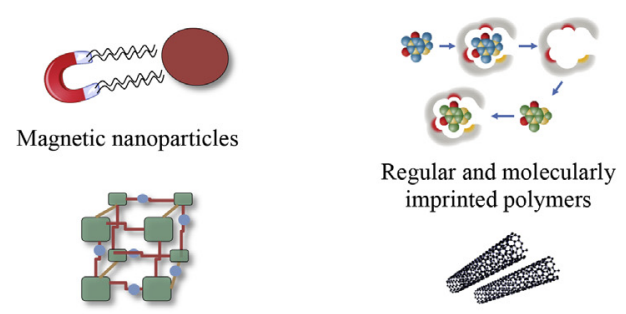

Metal-organic frameworks
Regular and molecularly imprinted polymers

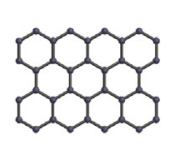

Graphene oxide

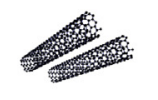

Carbon nanotubes

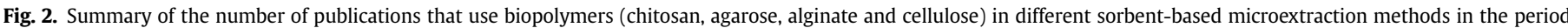
2014-2019, together with the main formats/devices of the sorbents, and main materials used in combination with biopolymers in the sorbents.

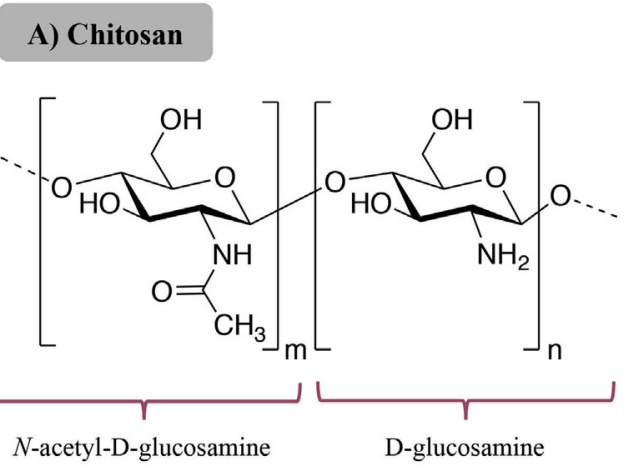

C) Alginate

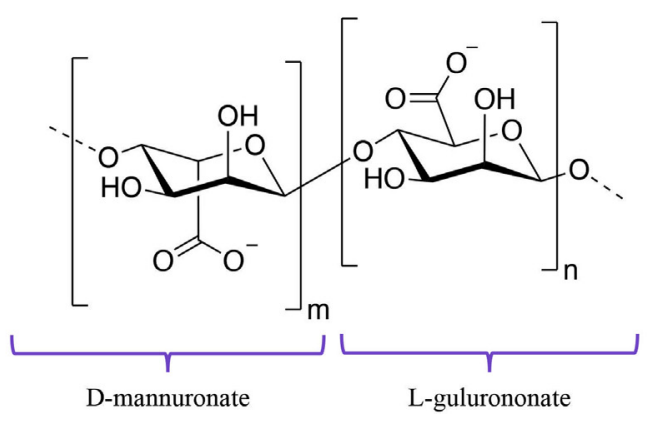

\section{B) Cellulose}

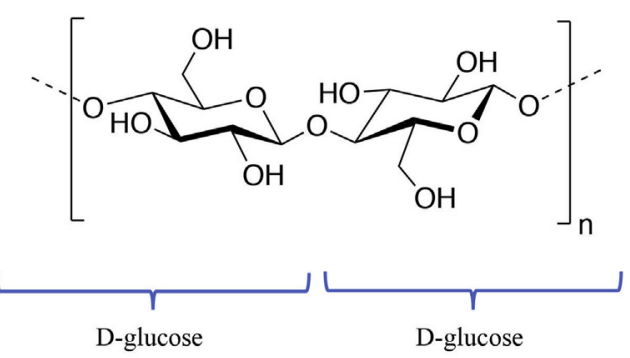

D) Agarose

Fig. 3. Structures of the biopolymers most frequently used in solid-based microextraction methods. 
chitosan fibers hydrothermally treated with different contents of acetic acid and then packed in glass pipettes. It is also interesting to mention the microchip reported by Gan et al. [37]. The extraction device consisted of a disk-shaped filter paper modified with chitosan by impregnation in a solution of the biopolymer, which was then placed on a thermoplastic microchip, but the extraction procedure involved the typical $\mu$-SPE steps: loading of the sample, washing and desorption.

Among hybrid chitosan-based sorbents for $\mu$-SPE (meaning by hybrid the incorporations of other materials than the biopolymer), carbonaceous materials are the most common materials used to prepare the composites, including multi-walled carbon nanotubes (MWCNTs) and GO. In the simplest strategy, both powdered materials are blended [38] or dispersed in an aqueous solution [39], and then packed to prepare the microextraction device. In any case, it results more common the addition of the carbon material to an acidic solution of chitosan, followed by insolubilization and formation of beads by the increase of the $\mathrm{pH}$ [40], or by the addition of a crosslinking agent (glutaraldehyde as the most common agent to promote it) to the mixture [41]. A similar approach has been described for the preparation of devices containing chitosan and other sorbent materials, such as a cartridge packed with chitosanmetal oxide nanoparticles [42] and the fabrication of a monolith sorbent based on chitosan, $\mathrm{GO}, \mathrm{C}_{18}$ and polypyrrole [43]. In several of the reported studies, chitosan was found to play an important role in the extraction efficiency of the developed device $[38,41]$, while in other cases it simply acted as dispersive matrix to confine the main extraction sorbent $[40,43]$.

Recently, Asiabi et al. described a composite material consisting of chitosan nanofibers and MOFs, prepared by electrospinning of an acidic solution containing chitosan and the MOF powder [44,45]. This procedure led to the preparation of spiderweb-like formed by electrospun nanofibers, presenting an enhanced surface area compared to the individual components. Both composites, with MOF MIL-68(Al) [44] and MIL-101(Fe) [45], were packed into filter disks to perform $\mu$-SPE. Chitosan powder has also been impregnated with an IL solution and then lyophilized to prepare a more efficient sorbent for the fabrication of a $\mu$-SPE cartridge [46].

Regarding $\mu$-dSPE, the use of chitosan has also been quite useful due to the possibility of preparing chitosan-based sorbents with different forms that favors its dispersion. Neat chitosan powder has been used as sorbent in acidic aqueous extracts, taking advantage of its cationic structure to enhance the extraction of negatively charged analytes [47]. Beads or microspheres of chitosan have been prepared by adding dropwise the acidic solution of chitosan to a $\mathrm{NaOH}$ solution $(\sim 1 \mathrm{M})$ using a syringe, followed by its further crosslinking [48] or combination with GO [49]. Then, they were confined in a polypropylene envelope to perform the $\mu$-dSPE method. Such envelope facilitates the further separation of the sorbent during the extraction and desorption steps. Chitosan beads have also been prepared using more sophisticated methods, such as sol-gel transition by conventional emulsion using epichlorohydrin as crosslinker [50] or ionic gelation using sodium tripolyphosphate [51]. In these cases, the beads are directly dispersed in the sample solution and separated by centrifugation or filtration.

The combination of chitosan with other materials has led to the development of interesting hybrid sorbents for $\mu$-dSPE. Chitosan has been grafted with polyaniline to obtain a composite with a rough surface, thus ensuring the presence of more pores to improve the retention of target analytes [52]. In other study, the surface of MWCNTs was modified with chitosan by dispersing the carbon material in an acidic solution of the biopolymer [53]. In both cases, the powder of the composite was dispersed in the aqueous sample to accomplish the $\mu$-dSPE process. Li et al. proposed the use of porous MOF/chitosan foams in $\mu$-dSPE, which were easily dispersed in the sample during extraction by ultrasounds, and then separated from the aqueous sample using tweezers to subsequently perform the elution step [54]. For the preparation of the foam, the MOF powder was dispersed in an acid solution containing chitosan, glutaraldehyde as crosslinking agent, and a gelatin, and afterwards the mixture was placed in a mold and freeze-dried to ensure formation the foam.

The miniaturized versions of MSPD methods have also benefited from the incorporation of chitosan as dispersant [55,56]. In these strategies, the chitosan powder and the solid sample are mixed using a pestle to accomplish the extraction of the compounds.

With respect to the use of chitosan in $\mathrm{m}-\mu$-dSPE, a high number of studies has been reported in the recent years compared with the remaining sorbent-based microextraction methods. All these applications involve the use of ferrite MNPs to obtain magnetic chitosan-based sorbents. The preparation of these magnetic sorbents can follow one of this main routes: i) the coprecipitation method, which consists of adding dropwise a $\mathrm{NaOH}(1-2.5 \mathrm{M})$ or $\mathrm{NH}_{3}$ solution ( $\left.25 \%, \mathrm{w} / \mathrm{w}\right)$ to an acidic solution containing $\mathrm{Fe}(\mathrm{II})$ and $\mathrm{Fe}(\mathrm{III})$ salts, and dissolved chitosan [57-71], or ii) the addition of the previously synthesized MNPs to an acidic solution of chitosan, followed by the crosslinking of the biopolymer using glutaraldehyde [72-83]. Other synthetic approaches include the chemical reduction of $\mathrm{Fe}(\mathrm{III})$ in presence of chitosan [84], the dispersion of modified-MNPs in an acidic solution of chitosan [85-87], or the use of (3-glycidyloxypropyl) trimethoxysilane [88] or sodium tripolyphosphate [71] as crosslinking agents. The functionalization of the MNPs with silica by the sol-gel method prior to the incorporation of chitosan has also been reported to improve the stability of the resulting composite $[74,85]$. Among other strategies that have been reported to prepare chitosan-based magnetic sorbents, it is interesting to mention the fabrication of stir beads composed of chitosan and polypyrrole in absence of any magnetic material [89]. In this case, the composite was prepared by crosslinking the chitosan with glutaraldehyde in presence of polypyrrole, while using a template to obtain the spherical shape. Then, a steel wire was inserted in the beads to obtain the magnetic sorbent, as shown in Fig. 4 (A). In the study of Xiao et al. [90], the powder of chitosan was dispersed in the aqueous sample followed by the addition of the previously prepared MNPs instead of preparing the magnetic composite. Both materials interacted due to electrostatic interactions, and the chitosan could be captured by the MNPs to perform the m- $\mu$-dSPE method.

The use of the neat chitosan-coated MNPs, in which the chitosan is the only material responsible for the extraction of the target analyte, has been scarcely reported $[65,67,72-74,76,85,90]$. In several of these studies, the chitosan was functionalized with specific groups, such as dithizone [76] and diphenyl diselenide [67] to enhance the extraction capability of the sorbent.

In most applications, magnetic chitosan sorbents were combined with other materials to prepare hybrid composites, such as GO [75,77,79-81,83,86,91] MIPs [60,63,69,70,78,80,81,88,92]; other polymers including polyaniline $[59,66,68]$, polypyrrole [57,61,89], and polythiophene [62,64]; and even ILs [77], deep eutectic solvents [69,82], surfactants [71], and antibodies [84]. When dealing with magnetic $\mathrm{GO} /$ chitosan sorbents, the $\mathrm{GO}$ is added to the reaction solution during the crosslinking of chitosan in presence of the MNPs. In the studies that incorporated imprinted or conventional polymers in the hybrid composite, the chitosanmodified MNPs prepared by any of the abovementioned methods are added to the polymerization solution. In the case of liquid additives, the magnetic chitosan is simply immersed in the liquid to ensure impregnation of its surface. Therefore, in most of these sorbents, the chitosan acts as protective layer to improve the stability of the MNPs while avoid their aggregation, or as matrix to 

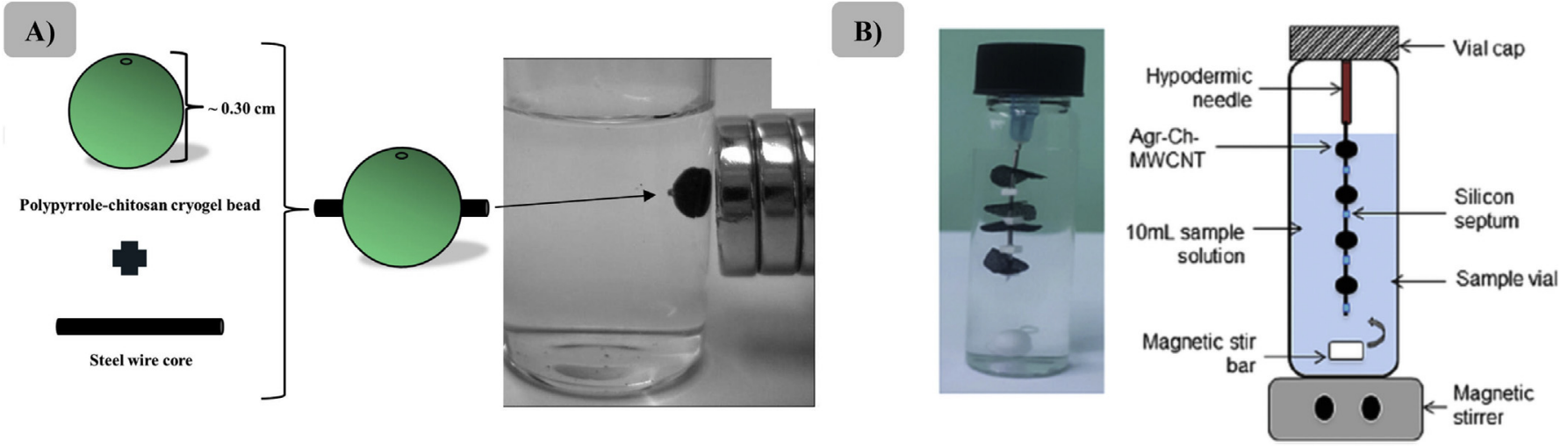

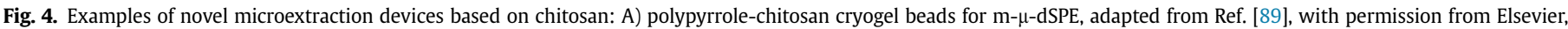
2017; and B) agarose-chitosan-MWCNTs films for TFME/STE, adapted from Ref. [94], with permission from Wiley, 2018.

homogenously disperse other materials in the resulting hybrid composite, rather than participating actively as main extraction agent in the microextraction process. Furthermore, all these preparation methods yield a heterogeneous composite, usually a polymeric matrix in which the MNPs are embedded. Main issue associated with heterogeneous composites link to inter-batch reproducibility issues. However, some authors have reported the formation of core-shell type particles, in which the MNP is perfectly coated with a layer of chitosan-based material [70,71,77,92].

Regarding SPME devices, chitosan-based coatings have been explored mainly in the TFME/STE configuration. This can be linked to the relatively easy fabrication of thin membranes composed of this biopolymer. In all reported studies, other materials besides chitosan were incorporated in the thin film to improve its mechanical stability and sorption capacity, including MWCNTs [93,94], Ag nanoparticles [95,96], agarose [94,97], $C_{18}$ [97], and halloysite nanotubes [98]. The most common method to prepare these devices consisted of blending a chitosan solution and a dispersion of the additional material, placing the mixture in a thin template, and then drying the mixture in an oven to evaporate the solvent. In the case of the chitosan/MWCNTs membrane prepared by Ge et al. [93], the surface of MWCNTs was modified by crosslinking the chitosan using glutaraldehyde, followed by immersion of a thin polypropylene membrane in a dispersion of such composite containing chitosan/MWCNTs. In all these studies, the membranes were freely dispersed all over the sample, or hold using a cotter pin, during the extraction step. Afterwards, membranes containing trapped analytes are removed using tweezers to accomplish the desorption step, being the exception the method reported by Wan Ibrahim et al. [94,97]. In this particular case, $4-5$ pieces of the prepared thin films were pierced using a syringe needle, and silicon septa were placed between each film to avoid them to detach, as it can be observed in Fig. 4 (B). This modified needle was immersed in the aqueous solution to perform the microextraction procedure, resembling the direct immersion mode of SPME (DI-SPME) using conventional fibers.

Composites containing chitosan have also been used in other SPME geometries. A SPME fiber was prepared using fused silica as support and a $\mathrm{ZnO}$ nanorods/chitosan composite as stationary phase [99]. The nanorods were in situ synthesized in a chitosan gel, where the fused silica fiber was immersed to form the coating. Then, the biopolymer immobilized on the fiber was crosslinked with glutaraldehyde to improve the stability of the coating, and used in DI-SPME followed by desorption using an organic solvent. Wu et al. reported the preparation of an in-tube SPME device taking advantage of the properties of chitosan [100]. In this study, the inner walls of polytetrafluoroethlyene tube were coated with polydopamine, followed by functionalization of this polymer with dialdehyde starch and chitosan by filling the tube with an ethanolic solution of both materials, followed by heating at high temperatures.

\subsubsection{Analytical applications with chitosan-based materials in sorbent-based microextraction}

Chitosan-based sorbents have been applied for the extraction of a wide variety of analytes from samples of quite different nature. Table 1 includes some representative applications of these sorbents in the different sorbent-based microextraction methods $[39,44,48,52,62,65,70,82,93,98,101-112]$. Given the diversity of reported applications, general trends will be discussed in the following section considering the nature of the analyte and the application area. As a general comment, applicable to all reported applications, it is important to highlight the following characteristics: low amounts of solid sorbents required, between 1 [49] and $500 \mathrm{mg}$ [47]; and relatively low volumes of samples, with average values of $10 \mathrm{~mL}$. In addition to this, it should also be noted that, despite the greenness properties of the biopolymer used and the environmental-friendliness associated to microextraction strategies, the reported applications still require the utilization of organic solvents (low amounts) to accomplish the desorption of the target analytes, while in most applications the biopolymers are commonly used with additional materials that are not always green.

The vast majority of the developed methods have been used for the extraction of organic compounds, covering from drugs and antibiotics $\quad[38,39,45,46,57,60,61,70,80,87,92,94]$, pesticides $[42,43,62,64,71,72]$, other persistent and emerging endocrine disruptor contaminants, to natural bioactive compounds $[41,53,55,56,69,86,90]$, and pigments [47,51]. Among the endocrine disruptor contaminants, polycyclic aromatic hydrocarbons (PAHs) [78,97], polychlorinated and polybrominated biphenyls (PCBs and PBBs) [49,58,93], phthalate acid esters (PAEs) [52,66,89], parabens [40,54], phenols [59,99], and other food contaminants [80,88], can be cited. All of the above-mentioned organic compounds have been determined in water samples coming from different sources $[40,42,48,49,54,58,60,62,64,71,78,93,94,98,99]$, or in aqueous extracts of food $[38,39,46,47,52,72,84,88]$, diluted drinks and beverages $[43,53,59,69,86,97]$, plants $[41,51,55,56]$, and dilutes or pretreated biological samples, such as urine, serum and blood $[45,57,61,70,80,87,90,92]$.

Most of the proposed extraction procedures have been coupled with LC for the determination of the target organic compounds, and using different detection systems depending on the nature of the 
Table 1

Main characteristics of representative analytical applications of biopolymers in sorbent-based microextraction methods.

\begin{tabular}{|c|c|c|c|c|c|c|c|c|}
\hline Method $^{\mathrm{a}}$ & $\begin{array}{l}\text { Additional material } \\
\text { in the composite }{ }^{a}\end{array}$ & Configuration & $\begin{array}{l}\text { Amount of } \\
\text { sorbent }\end{array}$ & Analytes $^{\mathrm{a}}$ (number) & Sample & $\begin{array}{l}\text { Analytical } \\
\text { technique }^{a}\end{array}$ & LOQ & Ref. \\
\hline \multicolumn{9}{|c|}{ Chitosan-based sorbents } \\
\hline$\mu$-SPE & GO & packed pipette tip & $4 \mathrm{mg}$ & sulfonamides (5) & eggs \& honey & LC-UV & $2.15-2.98 \mathrm{ng} \mathrm{g}^{-1}$ & [39] \\
\hline$\mu$-SPE & MIL-68(Al) & packed filter disk & $6 \mathrm{mg}$ & metals (2) & molluks & ICP-OES & $0.5 \mu \mathrm{g} \mathrm{L}^{-1}$ & [44] \\
\hline$\mu-\mathrm{dSPE}$ & - & $\begin{array}{l}\text { beads enclosed in an } \\
\text { envelope }\end{array}$ & $20 \mathrm{mg}$ & btex (5) & waters & GC-MS & $0.03-0.13 \mu \mathrm{g} \mathrm{L}^{-1}$ & [48] \\
\hline$\mu$-dSPE & polyaniline & composite powder & $20 \mathrm{mg}$ & PAEs (3) & milk & LC-UV & $0.4-0.5 \mu \mathrm{g} \mathrm{L}^{-1 \mathrm{~b}}$ & [52] \\
\hline $\mathrm{m}-\mu-\mathrm{dSPE}$ & $\mathrm{Fe}_{3} \mathrm{O}_{4} \mathrm{MNPs}$ & magnetic composite gel & $25 \mathrm{mg}$ & $\mathrm{Cd}$ & plants & FAAS & $0.68 \mu \mathrm{g} \mathrm{L}^{-1}$ & [65] \\
\hline$m-\mu-d S P E$ & $\begin{array}{l}\mathrm{Fe}_{3} \mathrm{O}_{4} \text { MNPs, } \\
\text { MWCNTs \& DES }\end{array}$ & $\begin{array}{l}\text { magnetic composite } \\
\text { powder }\end{array}$ & $8 \mathrm{mg}$ & DNA & blood & $\mathrm{UV}-\mathrm{Vis}$ & - & [82] \\
\hline $\mathrm{m}-\mu$-dSPE & MNPs \& MIP & $\begin{array}{l}\text { magnetic composite } \\
\text { powder }\end{array}$ & $25 \mathrm{mg}$ & drug (1) & serum \& urine & LC-DAD & $3.2-29.1 \mu \mathrm{g} \mathrm{L}^{-1}$ & [70] \\
\hline $\mathrm{m}-\mu$-dSPE & $\begin{array}{l}\mathrm{Fe}_{3} \mathrm{O}_{4} \mathrm{MNP} \& \\
\text { polythiophene }\end{array}$ & $\begin{array}{l}\text { magnetic composite } \\
\text { powder }\end{array}$ & $40 \mathrm{mg}$ & herbicides (3) & waters & GC-MS & $100 \mathrm{ng} \mathrm{L}^{-1}$ & [62] \\
\hline TFME/STE & MWCNTs & film $(1 \mathrm{~cm} \times 0.4 \mathrm{~cm} \times$ & 1 film & PBBs \& PCBs & waters & GC-MS & $0.12-0.6 \mathrm{ng} \mathrm{L}^{-1 b}$ & [93] \\
\hline TFME/STE & halloysite nanotubes & $\begin{array}{l}\text { film } \\
(1 \mathrm{~cm} \times 2 \mathrm{~cm} \times \sim 15 \mu \mathrm{m})\end{array}$ & 1 film & hormones (1) & waters & LC-FD & $1 \mu \mathrm{g} \mathrm{L}^{-1}$ & [98] \\
\hline \multicolumn{9}{|c|}{ Cellulose-based sorbents } \\
\hline$\mu$-SPE & $\beta$-cyclodextrin & packed microcolumn & $20 \mathrm{mg}$ & danofloxacin & milk & FD & $8.1 \mu \mathrm{g} \mathrm{L}^{-1}$ & [101] \\
\hline$\mu$-dSPE & Triton X-100 & $\begin{array}{l}\text { microcrystalline } \\
\text { cellulose powder }\end{array}$ & $5 \mathrm{mg}$ & $\mathrm{Cr}$ & waters & AAS & $6 \mu \mathrm{g} \mathrm{L}^{-1 \mathrm{~b}}$ & [102] \\
\hline $\mathrm{m}-\mu-\mathrm{dSPE}$ & $\mathrm{NiMn}_{2} \mathrm{O}_{4} \mathrm{MNPs}$ & $\begin{array}{l}\text { magnetic composite } \\
\text { powder }\end{array}$ & $30 \mathrm{mg}$ & BPs (6) & $\begin{array}{l}\text { waters \& plastic } \\
\text { food container }\end{array}$ & LC-UV & $1.85-2.76 \mu \mathrm{g} \mathrm{L}^{-1}$ & [103] \\
\hline TFME/STE & polydopamine & film $(1 \mathrm{~cm} \times 2 \mathrm{~cm} \times-)$ & 1 film & nitrophenols (2) & vegetable oils & LC-UV & $1.54-2.16 \mathrm{ng} \mathrm{L}^{-1 \mathrm{~b}}$ & [104] \\
\hline FPSE & PEG & $\underset{-)}{\text { film }}(2.5 \mathrm{~cm} \times 2 \mathrm{~cm} \times$ & 1 film & amphenicols (3) & milk & LC-UV & $55.9-58.9 \mu \mathrm{g} \mathrm{g}^{-1 \mathrm{~b}}$ & [105] \\
\hline FPSE & Carbowax $20 \mathrm{M}$ & film $(-x-x-)$ & 1 film & pesticides (4) & vegetables & GC-MS & $0.108-0.399 \mathrm{ng} \mathrm{g}^{-1}$ & [106] \\
\hline \multicolumn{9}{|c|}{ Alginate-based sorbents } \\
\hline$\mu-S P E$ & metal hydroxides & monolithic cartridge & - & $\mathrm{Pb}(\mathrm{II})$ & beverages & FAAS & $1.31 \mu \mathrm{g} \mathrm{L}^{-1}$ & [107] \\
\hline$\mu$-dSPE & MWCNTs & beads & $100 \mathrm{mg}$ & PAHs (3) & waters & GC-FID & $0.73-1.38 \mu \mathrm{g} \mathrm{L}^{-1}$ & [108] \\
\hline $\mathrm{m}-\mu$-dSPE & MNPs \& polypyrrole & magnetic beads & $300 \mathrm{mg}$ & $\begin{array}{l}\text { endocrine-disrupting } \\
\text { compounds ( } 3 \text { ) }\end{array}$ & waters & LC-FD & $2 \mu \mathrm{g} \mathrm{L}^{-1}$ & [109] \\
\hline \multicolumn{9}{|c|}{ Agarose-based sorbents } \\
\hline$\mu$-dSPE & MWCNTs & $\begin{array}{l}\text { composite gel cubes } \\
(3 \mathrm{~cm} \times 3 \mathrm{~cm} \times 3 \mathrm{~cm})\end{array}$ & - & herbicides (2) & water & GC-MS & $\begin{array}{l}1.064- \\
1.136 \mu \mathrm{g} \mathrm{L}^{-1}\end{array}$ & [110] \\
\hline $\mathrm{m}-\mu$-dSPE & $\mathrm{Fe}_{3} \mathrm{O}_{4} \mathrm{MNPs}$ & $\begin{array}{l}\text { magnetic composite } \\
\text { suspension }\end{array}$ & $\begin{array}{l}200 \mu \mathrm{L} \\
(20 \%, \mathrm{w} / \mathrm{v})\end{array}$ & Mo & beans & GFAAS & $49 \mathrm{ng} \mathrm{L}^{-1 \mathrm{~b}}$ & [111] \\
\hline TFME/STE & $\begin{array}{l}\text { molecularly imprinted } \\
\text { silica gel }\end{array}$ & $\begin{array}{l}\text { film } \\
(1 \mathrm{~cm} \times 1 \mathrm{~cm} \times 1 \mathrm{~mm})\end{array}$ & 1 film & sulfonamides (3) & waters & LC-UV & $0.20-0.56 \mu \mathrm{g} \mathrm{L}^{-1}$ & [112] \\
\hline
\end{tabular}

${ }^{a}$ For the definition of the abbreviations, refer to the list of abbreviations.

b LOD. LOQ was not reported.

analytes [38-43,45-47,51-56,59-61,69-71,84,87,89,90,92,94, 97-99]. The high number of couplings with LC is due to the compatibility of the solvent used in the desorption step with the chromatographic systems, with methanol and acetonitrile the most common. Nevertheless, several of the analytical applications have been performed in GC $[48,49,58,62,64,66,72,78,86,93]$. In these cases, evaporation and reconstitution of the organic solvents used in the desorption step are additional steps required, in order to obtain a final extract in a GC-compatible solvent.

Apart from the high number of applications devoted to organics, several of the proposed methods have also been applied to the determination of metals in water samples or in aqueous extracts, mainly using $\mathrm{m}-\mu$-dSPE $[63,65,67,74-76,79,81,91]$, but also in $\mu$-SPE involving a MOF/chitosan composite [44] or in TFME/STE with a chitosan membrane containing Ag nanoparticles [95]. It is worth mentioning that in this latter TFME/STE application, the thin film with the extracted metal ions was dissolved in nitric acid solution and analyzed by inductively coupled plasma mass spectrometry (ICP-MS), instead of carrying out a desorption step, thus avoiding reusing of the device. In the remaining methods, the metal ions were desorbed from the chitosan sorbent using acidic solutions. Furthermore, several of these methods were applied to the analysis of certified reference materials, thus demonstrating the applicability of the proposed methods with adequate results in comparison with conventional approaches $[74,76,95]$.

It is important to highlight the ability of chitosan-based sorbents to extract DNA with high purity from complex matrixes, such as blood and saliva, using m- $\mu$-dSPE methods [68,82,85], as well as using the $\mu$-SPE microchip prepared with a filter paper modified with chitosan [37]. These methods were combined with UV spectroscopy [82,85] or the polymerase chain reaction [37,78] for the quantification of the DNA. Other interesting applications include the use of mesoporous crosslinked chitosan microspheres in $\mu$ dSPE followed by LC and tandem mass spectrometry (MS/MS) for the isolation of $\mathrm{N}$-glycopeptides from biological matrixes [50], while a reusable magnetic composite composed of MNPs, chitosan and GO impregnated with an IL has been evaluated for the extraction of different proteins [77]. Regarding other bioclinical applications, the polydopamine in-tube SPME device modified with chitosan was successfully used in the determination of aldehydes and ketones liver cancer biomarkers in human blood, using LC and diode array detection (DAD) [100]

It is also interesting to mention that chitosan-based materials have been used not only as extraction sorbent but for the removal of interferences in clean-up steps based on $\mu$-dSPE $[113,114]$ and $\mathrm{m}-\mu$ dSPE $[73,83]$ when analyzing food samples. It is worth mentioning some specific applications, able to deal with complex samples, and 
comprising challenging analytical problems in different industrial areas. In this sense, magnetic chitosan-based sorbents have been used for the determination of PAEs in samples such as diapers [66] and saliva in contact with baby teether [89], with the aim of assessing the migration of these contaminants from the plastic products or containers. Another complex application reported a $\mu$ SPE device packed with neat chitosan fibers for the extraction of petroleum acids from crude oils before their determination by twodimensional gas chromatography $(\mathrm{GC} \times \mathrm{GC})$ and mass spectrometry (MS) with satisfactory results [36]. In another study, authors used chitosan-coated MNPs functionalized with a specific antibody to develop a selective method for the determination of aflatoxins in foodstuffs [84].

\subsection{Cellulose}

\subsubsection{Cellulose nature and uses}

Cellulose is the most abundant biopolymer on earth [115], being a fundamental component not only in plants but also in a large number of living species. As shown in Fig. 3, it is a linear polymer where 2 D-glucose units are linked by $\beta-1-4$ glycosidic bonds. Its structure contains many hydroxyl groups, resulting in a hydrophilic surface with numerous possibilities of chemical modifications. Besides its huge abundance, cellulose presents different interesting properties, including low cost, biodegradability, large surface area, and inertness. All these features have made this biopolymer very useful as drug carrier, flocculant or support in many different applications, such as in cosmetic, pharmaceutical and food areas [116].

\subsubsection{Cellulose in sorbent-based microextraction methods}

Recently, cellulose has received much attention in the extraction research field due to its high adsorptive capacity and biodegradability, and their use in this area has been recently reviewed $[30,33]$. Apart from the cellulose polymer, several of its derivatives have been particularly useful in this area. Cellulose acetate is the most studied cellulose derivative, in which the hydroxyl groups are replaced with acetate groups, leading to the preparation of a versatile material that can be used as membrane, powder, fiber or hydrogel. Nanocellulose is the term used to refer to nanometerscale cellulose fibril, which exhibits larger surface area and a higher number of hydroxyl groups. Microcrystalline cellulose, prepared by the acid hydrolysis of cellulose, serves as an excellent support thanks to its low density, insolubility in water and good mechanical properties [117].

Regarding $\mu$-SPE devices, different forms of cellulose have been explored. Ruiz-Palomero et al. functionalized nanocellulose with amino groups, which were used to covalently bond $\beta$-cyclodextrins to the surface of the biopolymer [101]. This modification of the nanocellulose provided nanocavities with selective recognition ability towards danofloxacin antibiotic, and the sorbent could even be reused at least 40 times. In another study, $\mathrm{CoFe}_{2} \mathrm{O}_{4}$ nanoparticles prepared by the co-precipitation method were coated with commercial cotton cellulose [118]. In both cases, few mg of the sorbent were packed in microcolumns and then used in an offline automated $\mu$-SPE strategy. Aqda et al. proposed a cellulose-based sorbent packed in a small cartridge for the development of an online $\mu$ SPE approach by replacing the loop of a LC valve by the $\mu$-SPE device [119]. In this case, cellulose triacetate fibers were synthesized by electrospinning, exhibiting different morphology and porosity depending on the solvent used for their preparation. The fibers prepared with acetone and dichloromethane showed higher surface area and led to better extraction performance.

In $\mu$-dSPE applications, microcrystalline cellulose has been used to prepare highly efficient sorbents. In all cases, the sorbent has been used in combination with surfactants to enhance the retention of the analytes. In the study reported by Cao et al. [120], a micellar-IL extraction was carried out to extract the target compounds from propolis, and then microcrystalline cellulose was added to trap and isolate the IL with the analytes. Finally, the resulting mixture was filtered using a nylon filter and the analytes were desorbed with an organic solvent. In a similar approach, sulfonated nanocellulose was used to trap the surfactant previously added to the sample for the extraction of $\mathrm{Ag}$ nanoparticles [121]. This sorbent was prepared by treating microcrystalline cellulose with sulfuric acid. This functionalization provided a negatively charged surface that easily interacts with the cationic surfactant. In the study reported by López-García et al., commercial microcrystalline cellulose was directly dispersed in the sample for the selective determination of $\mathrm{Cr}$ (III) [102]. Then, the surfactant Triton X-100 was added to the sample to perform a cloud point extraction, thus facilitating the collection of the solid phase. The use of low amounts (few $\mathrm{mg}$ ) of neat microcrystalline cellulose has been reported in the miniaturized version of MSPD to develop a greener and faster strategy compared to conventional methods, for the extraction of natural compounds from plant materials [122].

With regards to the preparation of magnetic sorbents for $m-\mu$ dSPE, MNPs of different nature have been combined with cellulose derivatives. $\mathrm{NiMn}_{2} \mathrm{O}_{4}$ MNPs were synthesized by a hydrothermal method in presence of cellulose fibers obtained from cotton wool. Finally, the sorbent is carbonized to obtain cellulose-based carbon fibers [103]. Following this strategy, $\mathrm{NiMn}_{2} \mathrm{O}_{4}$ grown on the surface of the fibers, leading to the preparation of a magnetic sorbent with a high surface area. Similarly, Abujaber et al. fabricated magnetic cellulose nanoparticles by a solvothermal method using microcrystalline cellulose swelled in solutions of $\mathrm{Fe}(\mathrm{II})$ and $\mathrm{Co}(\mathrm{II})$ salts [123]. These spheres were then coated with a hydrophobic IL, which was the main responsible material of the extraction of the target analytes. Ferrite MNPs have also been used in combination with cellulose to prepare a magnetic sorbent [124]. In this case, carboxymethyl cellulose was added to the reaction solution just after the synthesis of the MNPs by the co-precipitation method. These cellulose MNPs were then coated with a molecularly imprinted polymer to obtain a highly specific sorbent for the extraction of a plant hormone, with the cellulose coating acting as protective layer of the MNP. In these applications, desorption, evaporation and reconstitution steps were required for the determination of the analytes.

Certainly, cellulose have been more popular in TFME/STE, and particularly in FSPE applications, for which the number of studies reported have increased in the recent years (Fig. 2). Different strategies have been followed to use cellulose to prepare thin membranes for TFME/STE. Furthermore, in a number of applications, several films were added to the sample to increase the amount of sorbent (without sacrifying the thickness of the film) and improve the extraction efficiency $[18,125,126]$. Meng et al. employed neat cellulose filter papers ( 3 films) for the easy and lowcost extraction of a biomarker, taking advantage of their large surface area and the formation of hydrogen bonds with the analyte [125]. As it has been pointed out in the application of cellulose in other sorbent-phase microextraction methods, this biopolymer is very versatile and could be used in combination with different types of materials to obtain efficient hybrids materials. In this sense, cellulose filter papers have been easily modified with polydopamine by performing the polymerization in the presence of the filter paper [104], and with $\mathrm{ZrO}_{2}$ by depositing $\mathrm{Zr}$ gel films on the surface using a layer-by-layer sol-gel methodology [127]. An anticodeine aptamer has also been immobilized on aldehyde-modified cellulose filter papers to obtain thin films [128]. In these studies, the main role of the cellulose was to act as a support of the main 
extraction material. In a similar way, cellulose has been used as polymeric matrix of the thin film to disperse the solid sorbent: MWCNTs together with graphene [129] and $C_{18}$ [126]. For the preparation of these films, briefly, the solid materials were dispersed in a solution of cellulose acetate and placed on a flat surface, and then the solvent was evaporated for the solidification of the polymeric membrane. These tapes took advantage of the mechanical robustness of cellulose and the extraction capability of the solid materials.

Cellulose undoubtedly finds many applications in FPSE (Fig. 2), a variant of TFME/STE, in which a sol-gel derived sorbent is dispersed in an ultra-thin film fabric substrate, as shown in Fig. 5 (A) [20]. This new technique, developed by Kabir and Furton [130], benefits from the porosity, flexibility and permeability of the fabric support, together with the extraction capability of sorbent prepared by the sol-gel technique. This way, it is possible to perform simple, green, low cost, and fast analyses. FPSE is also a versatile technique since the choice of the fabric surface and the sorbent material directly influences the selectivity of the device. In this sense, polyester is used for the preparation of non-polar fabric phases, while cellulose substrate covers a wide range of polarity. It is important to highlight that in these applications the cellulose acts as support for the polymeric coating, thus it is not responsible of the extraction capability of the extraction device. Regarding the coating, polymers of different nature have been proposed, such as polydimethylsiloxane or $\mathrm{C}_{18}$ to fabricate non-polar media, and graphene or polyethylene glycol (PEG) to prepare more polar sorbents [20]. For the fabrication of the FSPE devices, the pretreated fabric substrate is immersed in a previously prepared sol solution and then dried to remove the solvent and to perform the condensation reaction that bonds the coating to the substrate. Among the possible coatings using fabric cellulose as substrate that can be prepared, PEG-based sorbents have been the most successful in FPSE applications for the extraction of highly polar compounds [105,131-134]. Carbowax 20 has also recently been used for the determination of polar compounds [106,135], while polytetrahydrofuran has been reported as a sorbent of medium polarity [136]. In most cases, these films can be reused at least 30 times, thus improving the greenness of the methodology $[106,131,133,134]$. In general, in the FPSE procedure, a single fabric film is dispersed in the sample with the aid of a magnetic stir bar. However, different approaches have been proposed to improve the extraction efficiency of the method. Roldán-Pijuán et al. together with the original inventors, reported a stir FPSE unit in which a magnetic stirring mechanism is integrated in the device using plastic cartridges and an iron wire, resembling a stir-cake sorptive extraction device [132]. More recently, a different configuration for FPSE was proposed by Pérez-Mayán et al. and the original authors [135]. In this case, three discs of the coated fabric substrate (4 mm in diameter) were vertically immersed in the sample using a stainless steel pin, in a way similar to DI-SPME.

SBSE has also benefited from the incorporation of cellulose to prepare reusable extraction devices. Abujaber et al. used in a SBSE application [137] the same type of cellulose MNPs material that was prepared for a previous $\mathrm{m}-\mu$-dSPE study [123]. In this case, instead of employing a direct dispersion of the magnetic material in the sample solution, the cellulose containing MNPs material was used to coat a stir bar, which could be reused for 5 consecutive extractions. During the stirring step, the MNPs were dispersed in the sample. Once the stirring was stopped, the MNPs rapidly returned to the stir bar, which facilitated their collection for the desorption step. In a totally different approach, a sol-gel coated cellulose fiber (using different polymers) was introduced in a polypropylene membrane [138]. This capsule was joined to another polypropylene membrane containing a magnet, as shown in Fig. 5 (B). This microextraction capsule device was stirred in the sample thanks to the magnetic component, under the typical extraction procedure for SBSE applications. The use of this sorbent allowed the direct and fast analysis of complex matrixes due to the polypropylene protective membrane and the autonomous-stirring features of the device, which could be reused up to 10 times.

\subsubsection{Analytical applications with cellulose-based materials in sorbent-based microextraction}

As shown in Table 1, cellulose-based sorbents are very versatile and have been employed in different analytical applications, without following a general trend depending on the microextraction approach. As a common feature for all these applications, low amounts (few mg) of the sorbent or a small piece of cellulose material were employed, together with low amounts of initial sample, thus improving the preconcentration of the method. As for chitosan-based sorbent-based extraction methods, the use of cellulose-based sorbents in microextraction required the desorption of the analytes (and cleaning before reusability) using an organic solvent or a buffer solution. Given the low thermal stability of this biopolymer, thermal desorption could not be used in these applications.
A)

Cellulose fabric
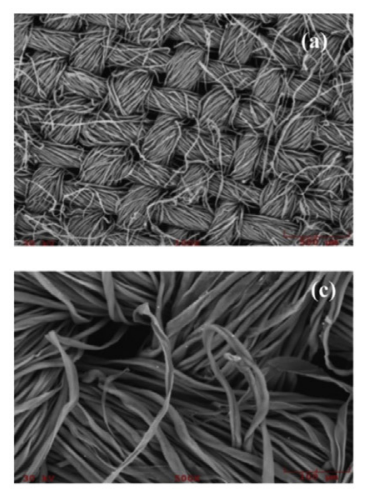

PEG-coated cellulose fabric
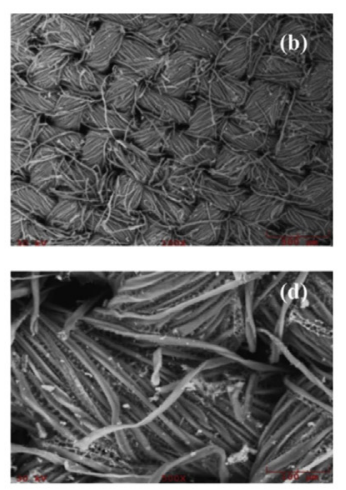

B)

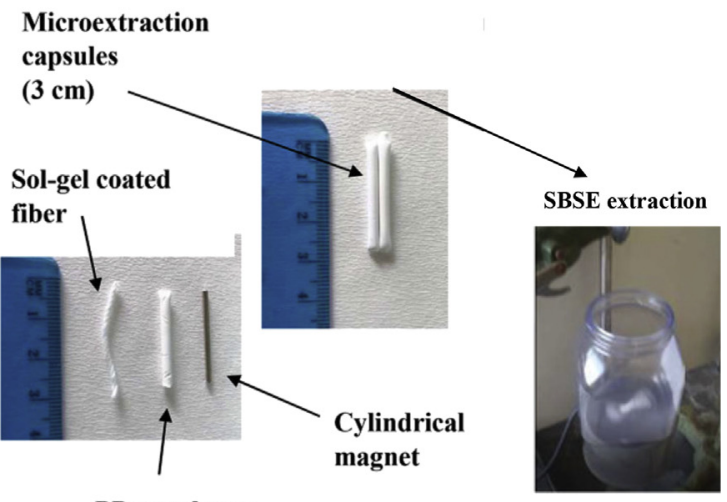

PP membrane

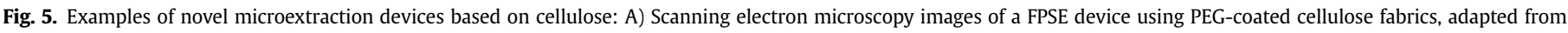
Ref. [105], with permission from Elsevier, 2015; and B) Encapsulated sol-gel coated cellulose fibers for SBSE, adapted from Ref. [138], with permission from Wiley, 2019. 
Most methods using cellulose-based sorbents were intended for the determination of organic compounds, including pharmaceuticals and antibiotics $[101,104,105,119,123,128,131,133,138]$, pesticides [106,132,134,135], plasticizers [103,126,137], and hormones [136]. The extraction of natural or bioactive compounds from plantderived materials has also been reported in several studies $[118,120,122,124]$. Cellulose-based sorbent have also being useful in bioclinical analysis for the extraction of nucleosides [127] and biomarkers [125]. The determination of metal species using these sorbents has been barely described [102,121].

The methods have been mainly applied for the analysis of environmental waters [102,103,123,126,129,131,134,136], body fluids, including urine and blood plasma [119,125,127,128,131], and beverages [101,105,133-135,137,138]. In the case of the analysis of solid samples, such as food and plants, the application of the sorbent-based microextraction method usually required a previous extraction step of the sample using an organic solvent, which may reduce the sustainability of the process [120,121,124]. However, it is interesting to mention the suitability of FPSE for the extraction of target analytes from complex matrices without requiring an exhaustive pretreatment of the sample, such as milk [105,133], juices [134], vegetables [106], and wine [135].

In the majority of the cases due to composition of the solvent used in the desorption step, the desorption solvent after applying the microextraction method is injected in a LC system coupled with UV-Visible, MS or fluorescence detection depending on the nature of the analytes [101,103-105,118-120,122-126,128,129, $131-133,135,136,138]$. Only a few studies reported the use of GC in combination with different detectors for the determination of the target compounds [106,134,137]. Others determination instruments have been used considering the nature of the extracted analytes, such as electrothermal AAS for Cr determination [102], capillary electrophoresis for Ag nanoparticles [121], fluorimetric determination of danofloxacin [101], or direct nanoelectrospray ionization-tandem mass spectrometry for nucleosides detection [127].

\subsection{Alginate}

\subsubsection{Alginate nature and uses}

Alginate is a biopolymer extracted from the cell walls of a large number of algae species, normally as sodium salt. This anionic polymer consists of linked residues of D-mannuronic acid (M-block) and L-guluronic acid (G-block), as it can be observed in Fig. 3. The physical properties of alginate depend on the distribution of $\mathrm{M}$ - and G-block units along the chain, which is strongly related to the natural source from which it is obtained. One of the most attractive features of alginate is its ability to form viscous and biocompatible hydrogels when it is crosslinked with different agents, with divalent cations the most common [139]. However, these ionically crosslinked materials suffer from certain stability issues in complex media, while hydrogels with a wide range of mechanical properties are obtained using covalent crosslinkers. Furthermore, grafting alginate hydrogels with other polymers or the functionalization of its structure with specific groups lead to the formation of stimuliresponsive materials, including $\mathrm{pH}$ and thermo-sensitive hydrogels [140]. Given all these characteristics, hydrogels prepared from alginate have been particularly useful in biomedical applications [139].

\subsubsection{Alginate in sorbent-based microextraction methods including analytical features and their applications}

More recently, several studies have reported the incorporation of this biopolymer in sorbent-based microextraction methods (Fig. 2) [32]. All reported applications of alginate in microextraction strategies used $\mathrm{CaCl}_{2}$ as crosslinker agent to prepare the alginate hydrogel. Furthermore, the alginate always serves as a matrix to disperse an additional solid material and to increase the surface area, this way improving the interaction of the target compounds with the sorbent. Table 1 includes representative examples. The preparation of the sorbent containing alginate is different depending on the sorbent-based approach, and also depending on the additional materials included in the sorbent.

Wang et al. [107] prepared an alginate monolithic sorbent containing layered double hydroxides nanosheets to develop a $\mu$ SPE device for the selective extraction and determination of $\mathrm{Pb}$ (II) in beverages using flame atomic absorption (FAAS). In this case, the nanosheets were hydrothermally prepared and dispersed in an aqueous solution of alginate. This mixture was added to a $\mathrm{CaCl}_{2}$ solution placed in an empty cartridge to prepare the monolithic column.

The incorporation of alginate in $\mu$-dSPE methods has been accomplished in different ways, as a function of the format in which the biopolymer was used. Thus, beads or spherical particles of alginate containing GO [141] or MWCNTs [108] have been prepared and directly used for the extraction of non-steroidal anti-inflammatory drugs and PAHs, respectively. The fabrication of the sorbent consists on mixing a dispersion of the carbonaceous material with a sodium alginate solution, followed by the dropwise addition of the previous mixture to a $\mathrm{CaCl}_{2}$ solution. The obtained beads are then cured in the metal solution, and washed with water to remove the excess of reagents. Depending on the characteristics of the needle and the distance needle-solution used during the dropwise addition step, beads of different sizes were obtained. In the microextraction procedure, around $100 \mathrm{mg}$ of the beads were dispersed in the sample to retain the analytes. Afterwards, analytes are desorbed from the beads using an adequate organic solvent and determined by LC [141] or GC [108].

Zare et al. reported the use of alginate fibers containing $\mathrm{Zr}$ nanoparticles in a $\mu$-dSPE approach for the extraction of pesticides from water and juice samples [142]. The preparation of the sorbent resembles that of the alginate-based beads with slight modifications. In this case, the dispersion of $\mathrm{Zr}$ nanoparticles in the sodium alginate solution was rapidly injected into the $\mathrm{CaCl}_{2}$ solution to obtain a tangled fiber as shown in Fig. 6 (A). The swelled fiber (100 mg) was added to the sample and dispersed with the aid of a magnetic stir bar. The sorbent was then easily separated from the sample using tweezers, and the desorption of the analytes was carried out by immersing the fiber in a small volume of organic solvent. The configuration of this sorbent provided enhanced surface area, which led to high recoveries of the analytes in real samples in a short time compared with other methods reported in the literature.

Most applications of alginate in $\mathrm{m}-\mu$-dSPE methods have been reported by Bunkoed et al. [109,143,144]. For the preparation of the magnetic sorbents, MNPs were firstly prepared by the coprecipitation method, and then dispersed in a sodium alginate solution. Afterwards, the mixture is dropwise added to the metal stock solution to obtain the magnetic beads. In the first study, MWCNTs were also included in the initial dispersion to prepare a composite with improved extraction ability towards PAHs [143]. In the following applications, the $\mathrm{Fe}_{3} \mathrm{O}_{4} /$ alginate beads were coated with a layer of polypyrrole [109] or polyaniline [144], by dispersing the magnetic beads in the monomer solution before the polymerization reaction. In all cases, low amounts (few $\mathrm{mg}$ ) of the magnetic sorbent were dispersed in the water samples to isolate the PAHs $[143,144]$ or endocrine-disrupting compounds [109]. Desorption of trapped analytes by the magnetic material is normally accomplished with acetonitrile, followed by evaporation and reconstitution prior to the LC-fluorescence detection analysis. It is interesting 

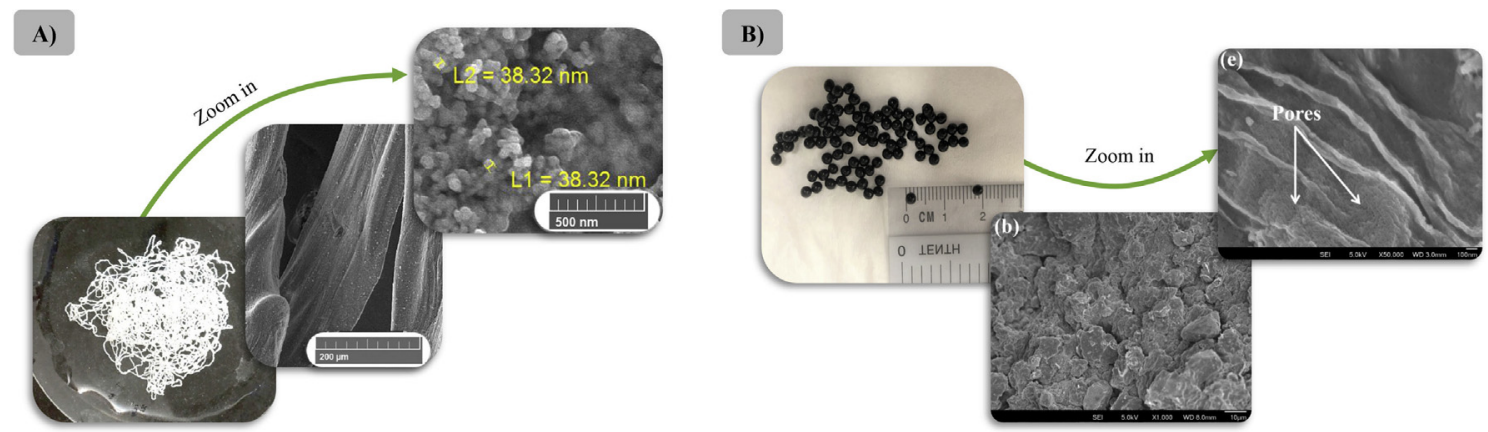

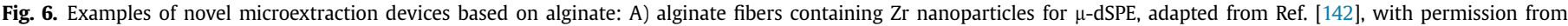
Elsevier, 2016; and B) magnetic $\mathrm{Fe}_{3} \mathrm{O}_{4} /$ alginate/MIL-101(Cr) beads for $\mathrm{m}-\mu$-dSPE, adapted from Ref. [145], with permission from Springer Nature, 2019.

to mention that, besides the advantages of the paramagnetic features of the sorbent, these beads could be reused from 6 [144] to 16 times [109], after proper washing with acetonitrile.

Recently, Tan et al. reported the use of a magnetic composite that included alginate beads and an amino-functionalized MOF MIL-101(Cr) [145]. Both the MNPs and the MOF were synthesized by the solvothermal method, dispersed in a sodium alginate solution, and added dropwise to a $\mathrm{CaCl}_{2}$ solution to obtain the composite beads with a diameter around $2.5 \mathrm{~mm}$, as shown in Fig. 6 (B). Authors demonstrated that the MOF was the main responsible in the extraction of polar herbicides from water samples, showing better performance compared to other conventional sorbents. This sorbent could be also reused in 10 consecutive extractions without any loss in its extraction capability.

Alginate hydrogels have also been used for the preparation of a SPME fiber that could be reused for at least 5 consecutive extractions in DI-SPME mode, followed by desorption in methanol [146]. The preparation of the fiber consisted of immersing a polypropylene hollow fiber in an aqueous solution containing sodium alginate and zein, a corn protein. After heating for a few hours, a stainless-steel wire was inserted in the lumen of the hollow fiber as fiber support, and the device was then immersed in a $\mathrm{CaCl}_{2}$ solution to accomplish the crosslinking of the alginate on the hollow fiber pores. The proposed device exhibited better results for the extraction of polar organic compounds, with the alginate hydrogel being the main component, thus playing an important role in the extraction capacity of the sorption phase rather than acting as a mere support.

\subsection{Agarose}

\subsubsection{Agarose nature and uses}

Agarose is a natural biopolymer extracted from seaweed. As shown in Fig. 3, this polysaccharide presents alternating D-galactose and 3,6-anhydro-L-galactose units in its structure, with a hydroxyl groups-rich surface, which leads to its characteristic inertness. For this reason, agarose usually requires functionalization in order to improve its reactivity. It also presents stability in a wide range of $\mathrm{pH}$ and temperature, hydrophily, flexibility and mechanical strength [31].

One of the most interesting properties of agarose is its gelling capacity, that facilitates the fabrication of agarose films. Agarose gels are suitable for diffusion and electrokinetic migration of compounds and can be easily modified to tune their physicochemical properties. Moreover, it is possible to incorporate other components in its structure during the gelation process, leading to the preparation of interesting hybrid materials [147]. Due to this set of features, agarose (mainly in its gel form) has been widely used in many research fields, such as biomedicine, food industry, immunology and separation science, including electrophoresis techniques and extraction methods $[32,148]$.

\subsubsection{Agarose in sorbent-based microextraction methods including analytical features and their applications}

Regarding the use of this biopolymer in extraction techniques, agarose particularly finds numerous applications in electromembrane extraction (EME), acting as a support of the extraction solvent or as an interface between the donor and acceptor phases [149]. These applications will not be covered in the present review since EME is classified as a liquid-phase extraction technique, considering the liquid nature of the extracting phase. With regards to sorbent-based microextraction methods, agarose has been scarcely used in the recent years compared with the previously discussed biopolymers (Fig. 2). In the reported applications, the main role of agarose gels is to act as support or as dispersion media of other solid materials to increase the surface area of the resulting sorbent, as shown in Table 1.

Sanagi et al. have reported the use of agarose to prepare sorbents for $\mu$-dSPE applications $[110,150]$. In one of the proposed methods, the biopolymer was grafted with poly(methyl methacrylate) following a microwave-assisted free radical copolymerization approach, in which agarose was mixed with the starting reagents required for the preparation of the polymer [150]. The resulting material was ground, and $80 \mathrm{mg}$ were used in the $\mu$-dSPE method for the extraction of $\mathrm{Cd}, \mathrm{Ni}, \mathrm{Cu}$ and $\mathrm{Zn}$ present in waters and digested vegetables samples, followed by determination using ICPMS. In a different strategy, authors incorporated MWCNTs into the agarose gel [110]. In this case, MWCNTs and agarose were mixed in different proportions under stirring, and then allowed to form the gel. The resulting material was cut in cubic pieces of $3 \times 3 \times 3 \mathrm{~mm}$ as shown in Fig. 7 (A), to obtain a sorbent that could be easily dispersed and manipulated during the $\mu$-dSPE procedure. The presence of MWCNTs increased the number of interaction sites, and therefore the amount added in the gel was significant for the efficient extraction of herbicides from waters. In the desorption step, tetrahydrofuran was used as desorption solvent and then injected in the GC-MS system for the determination of the analytes.

In $\mathrm{m}-\mu$-dSPE applications, agarose is combined with ferritebased MNPs to synthesize the magnetic sorbent [111,151,152]. In the method reported by Poursheikhi et al., the MNPs were prepared by the coprecipitation method in presence of agarose to obtain the agarose gel matrix containing MNPs [111]. In the remaining methods, the dispersion of the MNPs in the agarose gel was accomplished by a water-oil emulsion technique using Span-80 [152] or Span-85 [151] as the oil phase, and the aqueous agarose solution as the water phase. This procedure was carried out either 
A)
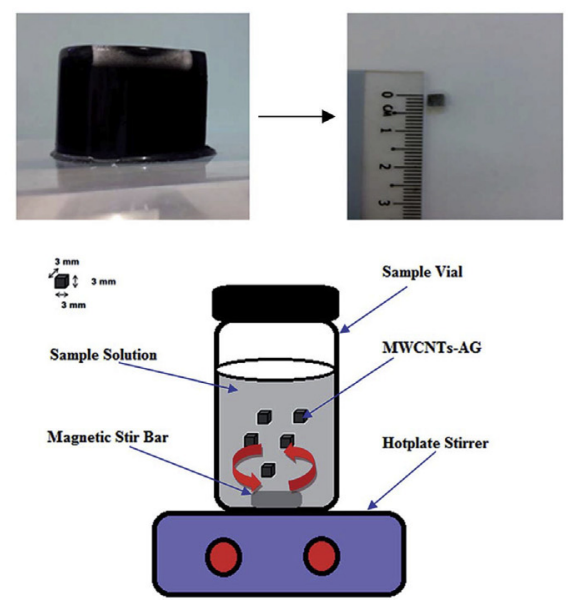

B)

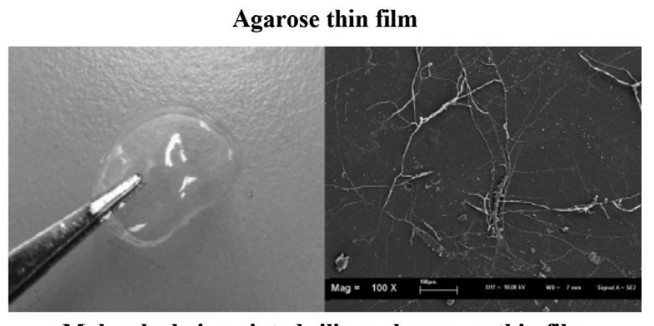

Molecularly imprinted silica gel-agarose thin film

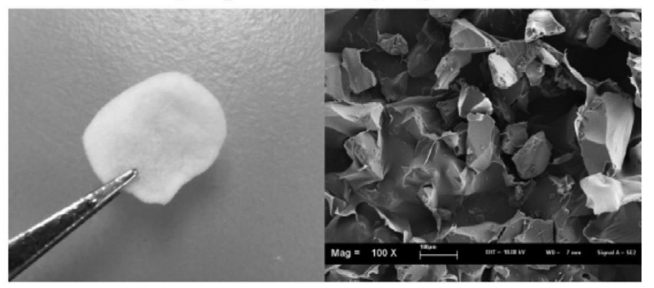

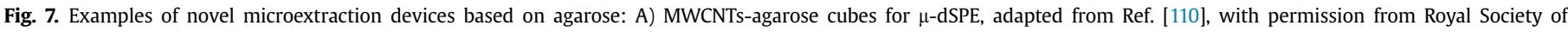
Chemistry, 2015; and B) neat agarose and molecularly imprinted silica gel-agarose membranes for TFME/STE, adapted from Ref. [112], with permission from Elsevier, 2019.

simultaneously during the synthesis of the MNPs by the coprecipitation method [151], or by adding the prepared core-shell MNPs@SiO ${ }_{2}$ particles to the water-oil mixture [152]. In all cases, the sorbent particles were subjected to an epoxy activation step, followed by their functionalization with the aim of improving the selectivity for the target compounds. The reported surface modifications included phenylephrine for the extraction of Mo from beans [111], a Schiff base ligand for the formation of complexes and extraction of $\mathrm{UO}_{2}$ (II) from waters [151], and an aptamer to selectively extract aflatoxins from maize samples [152]. Trapped analytes are desorbed from the magnetic materials using organic solvents, and subsequently determined by spectrophotometric techniques [111,151] or LC-fluorescence detection [152], depending on the nature of the analytes. It is interesting to mention the magnetic field agitation device reported by Hashemi et al. [111,151], which could be programmed to change the magnetic field around the sample cell in order to control the dispersion of the magnetic sorbent through the sample.

TFME/STE methods have also taken advantage of the gelation ability of agarose to form thin films. Molecularly imprinted silica gel [112] and $C_{18}$ [153] have been dispersed in warm agarose aqueous solutions and placed on a flat surface, followed by the evaporation of the sorbent to allow solidification of the gel, thus forming the films. Fig. 7 (B) shows the surface morphology of the agarose and molecularly imprinted silica gel-agarose thin films, which were used for the selective extraction of sulphonamides in waters due to the imprinting properties of the membranes [112]. In the case of the agarose films containing $\mathrm{C}_{18}$, the incorporation of the additional solid material to the agarose matrix led to the enhanced extraction of PAHs from coffee beverages [153]. The use of agarose as polymeric matrix is important since it ensures the preparation of an environmentally friendly film with a high mechanical stability. In both applications, the films were directly introduced in the sample to perform the extraction with the aid of a magnetic stir bar [112] or ultrasounds [153]. For the desorption step prior to the injection in the LC system, the films were immersed in a low volume of desorption solvent.

\section{Concluding remarks}

The current sorbent-based microextraction approaches that have been designed with the aim of enhancing the operational characteristics, environmentally friendliness and extraction performance of the analytical procedure have also taken advantage of the biodegradability and biocompatibility of biopolymers. Chitosan, cellulose, alginate and agarose are the most explored options among the existing biopolymers, with an increasing number of studies reported in the last 6 years. Their success mainly lies in the versatility of their physicochemical properties, and their flexibility of design and adaptation, which allow their use un numerous formats and configurations suitable for the different microextraction approaches, such as spherical particles, membranes, fibers, gels, and foams.

However, there is a still a need for a better understanding of the inherent physicochemical properties of these biomaterials to provide reliable and founded guidelines that allow the adequate selection of the most suitable biopolymer according to specific requirements. In general, the incorporation of these biopolymers in microextraction is based on their use as polymeric matrix with other solid materials (CNTs, GO, MNPs, MIPs, MOFs, etc.), with the purpose of improving the overall extraction capacity of the sorbent. Therefore, their main role in these applications is to serve as support or protection for other materials that are responsible of the extraction of the target compounds. In this sense, biopolymers seem to find a promising application in TFME/STE as flexible membranes, and clearly the use of cellulose as fabric substrate in FPSE is quite successful nowadays. In the same way, chitosan is the most adequate option in m- $\mu$-dSPE in combination with MNPs due to its crosslinking ability, that allows the preparation of stable magnetic sorbents.

In comparison with other methods reported in the literature, the use of these biopolymers to obtain extraction materials with enhanced surface area provides faster sample preparation strategies. In addition, the protection role of biopolymers in most of the extraction devices allows the analysis of complex matrices with satisfactory relative recovery values and even the reutilization of the sorbent. All these characteristics make biopolymers adequate candidates to prepare sorbents that may be easy to manipulate, reuse and incorporate in fast and simple microextraction strategies, which are very attractive features for their implementation in routine laboratories.

Considering the scope of the present review, future advances may be mainly focused on exploiting the gelling properties of these materials to prepare new microextraction devices with increasing surface area. The chemical functionalization of their surface still requires to be addressed to avoid the use of additional solid 
materials that may reduce the greenness of the sorbent. In this sense, it would be interesting the incorporation of thorough studies trying to elucidate the possible interactions between the analytes and the biopolymers to understand the mechanism behind the extraction process, which undoubtedly will help in the design of more useful biomaterials.

\section{CRediT author statement}

Idaira Pacheco-Fernández: Conceptualization, Investigation, Writing - original draft.

Diego W. Allgaier-Díaz: Investigation, Writing - original draft. Giulia Mastellone: Investigation, Writing - original draft.

Cecilia Cagliero: Writing - review \& editing.

David Díaz Díaz: Conceptualization, Writing - review \& editing. Verónica Pino: Conceptualization, Funding acquisition; Project administration; Resources; Supervision; Writing - review \& editing.

\section{Acknowledgements}

I.P.-F. thanks the Agencia Canaria de Investigación, Innovación y Sociedad de la Información (ACIISI), co-funded by the European Social Fund, for her FPI PhD fellowship. G.M. thanks the Erasmus + Programme. C.C. thanks the project financially supported by the 'Ricerca Locale' (Ex60\%2019) of the University of Turin, Turin (Italy). D.D.D. thanks the Spanish Ministry of Science, Innovation and Universities for the Senior "Beatriz Galindo" Distinguished Researcher Award. V.P. thanks the Spanish Ministry of Economy and Competitiveness (MINECO) for the Project Ref. MAT2017-89207-R. The authors extend their appreciation to the Spanish Ministry of Science, Innovation and Universities for granting the Spanish Network of Excellence in Sample Preparation (RED2018-102522-T). This article is based upon work from the Sample Preparation Task Force and Network supported by the Division of Analytical Chemistry of the European Chemical Society.

\section{References}

[1] A. Gałuszka, Z. Migaszewski, J. Namieśnik, The 12 principles of green analytical chemistry and the SIGNIFICANCE mnemonic of green analytical practices, Trends Anal. Chem. 50 (2013) 78-84.

[2] S. Armenta, S. Garrigues, F.A. Esteve-Turrillas, M. de la Guardia, Green extraction techniques in green analytical chemistry, Trends Anal. Chem. 116 (2019) 248-253.

[3] I. Pacheco-Fernández, V. Pino, Green solvents in analytical chemistry, Curr. Opin. Green Sustain. Chem. 18 (2019) 42-50.

[4] M. Rutkowska, J. Płotka-Wasylka, M. Sajid, V. Andruchc, Liquid-phase microextraction: a review of reviews, Microchem. J. 149 (2019) 103989.

[5] V. Jalili, A. Barkhordari, A. Ghiasvand, A comprehensive look at solid-phase microextraction technique: a review of reviews, Microchem. J. 152 (2019) 104319.

[6] C. Poole, Z. Mester, M. Miró, S. Pedersen-Bjergaard, J. Pawliszyn, Extraction for analytical scale sample preparation (IUPAC Technical Report), Pure Appl. Chem. 88 (2016) 649-687.

[7] E.V.S. Maciel, A.L. de Toffoli, E.S. Neto, C.E.D. Nazario, F.M. Lanças, New materials in sample preparation: recent advances and future trends, Trends Anal. Chem. 119 (2019) 115633.

[8] J. Płotka-Wasylka, N. Szczepańska, M. de la Guardia, J. Namieśnik, Miniaturized solid-phase extraction techniques, Trends Anal. Chem. 73 (2015) $19-38$.

[9] A. Andrade-Eiroa, M. Canle, V. Leroy-Cancellieri, V. Cerdà, Solid-phase extraction of organic compounds: a critical review (Part I), Trends Anal. Chem. 80 (2015) 641-654.

[10] E.M. Thurman, K. Snavely, Advances in solid-phase extraction disks for environmental chemistry, Trends Anal. Chem. 19 (2000) 18-26.

[11] A. Chisvert, S. Cárdenas, R. Lucena, Dispersive micro-solid phase extraction, Trends Anal. Chem. 112 (2019) 226-233.

[12] D. Wianowska, M. Gil, New insights into the application of MSPD in various fields of analytical chemistry, Trends Anal. Chem. 112 (2019) 29-51.

[13] A. Ríos, M. Zougagh, Recent advances in magnetic nanomaterials for improving analytical processes, Trends Anal. Chem. 84 (2016) 72-83.

[14] J. Pawliszyn, Handbook of Solid-phase Microextraction, first ed., Elsevier, Waltham, 2012.
[15] H. Piri-Moghadam, M.N. Alam, J. Pawliszyn, Review of geometries and coating materials in solid phase microextraction: opportunities, limitations, and future perspectives, Anal. Chim. Acta 984 (2017) 42-65.

[16] M.E. Costa Queiroz, I.D. de Souza, C. Marchioni, Current advances and applications of in-tube solid-phase microextraction, Trends Anal. Chem. 111 (2019) 261-278.

[17] F. David, N. Ochiai, P. Sandra, Two decades of stir bar sorptive extraction: a retrospective and future outlook, Trends Anal. Chem. 112 (2019) 102-111.

[18] Y.A. Olcer, M. Tascon, A.E. Eroglu, E. Boyacı, Thin film microextraction: towards faster and more sensitive microextraction, Trends Anal. Chem. 113 (2019) 93-101.

[19] S. Sisalli, A. Adao, M. Lebel, I. Le Fur, P. Sandra, Sorptive tape extraction - a novel sampling method for the in vivo study of skin, LC GC Eur. 19 (2006) 33-39.

[20] V. Kazantzi, A. Anthemidis, Fabric sol-gel phase sorptive extraction technique: a review, Separations 4 (2017) 20.

[21] Y. Chen, L. Xia, R. Liang, Z. Lu, L. Li, B. Huo, G. Li, Y. Hu, Advanced materials for sample preparation in recent decade, Trends Anal. Chem. 120 (2019) 115652.

[22] T. Zhou, G. Che, L. Ding, D. Sun, Y. Li, Recent progress of selective adsorbents: from preparation to complex sample pretreatment, Trends Anal. Chem. 121 (2019) 115678.

[23] E. Turiel, A. Martín-Esteban, Molecularly imprinted polymers-based microextraction techniques, Trends Anal. Chem. 118 (2019) 574-586.

[24] M.J. Trujillo-Rodríguez, H. Nan, M. Varona, M.N. Emaus, I.D. de Souza, J.L. Anderson, Advances of ionic liquids in analytical chemistry, Anal. Chem. 91 (2019) 505-531.

[25] A. Gutiérrez-Serpa, I. Pacheco-Fernández, J. Pasán, V. Pino, Metal-organic frameworks as key materials for solid-phase microextraction devices-a review, Separations 6 (2019) 47.

[26] P. Rocío-Bautista, P. González-Hernández, V. Pino, J. Pasán, A.M. Afonso, Metal-organic frameworks as novel sorbents in dispersive-based microextraction approaches, Trends Anal. Chem. 90 (2017) 114-134.

[27] L. Chen, Q. Wu, J. Gao, H. Li, S. Dong, X. Shi, L. Zhao, Applications of covalent organic frameworks in analytical chemistry, Trends Anal. Chem. 113 (2019) $182-193$.

[28] P. Rocío-Bautista, I. Taima-Mancera, J. Pasán, V. Pino, Metal-organic frameworks in green analytical chemistry, Separations 6 (2019) 33.

[29] D.F. do Amaral, V. Guerra, A.G.C. Motta, D. de Melo e Silva, T.L. Rocha, Ecotoxicity of nanomaterials in amphibians: a critical review, Sci. Total Environ. 686 (2019) 332-344.

[30] G. Mafra, M.T. García-Valverde, J. Millán-Santiago, E. Carasek, R. Lucena, S. Cárdenas, Returning to nature for the design of sorptive phases in solidphase microextraction, Separations 7 (2020) 2.

[31] M. Rinaudo, Main properties and current applications of some polysaccharides as biomaterials, Polym. Int. 57 (2008) 397-430.

[32] M.M. Sanagi, S.H. Loh, W.N.W. Ibrahim, N. Pourmand, A. Salisu, W.A.W. Ibrahim, I. Ali, Agarose- and alginate-based biopolymers for sample preparation: excellent green extraction tools for this century, J. Separ. Sci. 39 (2016) 1152-1159.

[33] J.-H. Wu, C.-Y. He, Advances in cellulose-based sorbents for extraction of pollutants in environmental samples, Chromatographia 82 (2019) $1151-1169$.

[34] M. Rinaudo, Chitin and chitosan: properties and applications, Prog. Polym. Sci. 31 (2006) 603-632.

[35] M. Dash, F. Chiellini, R.M. Ottenbrite, E. Chiellini, Chitosan-A versatile semisynthetic polymer in biomedical applications, Prog. Polym. Sci. 36 (2011) 981-1014.

[36] G.-T. Zhu, X.-L. Hu, S. He, X.-M. He, S.-K. Zhu, Y.-Q. Feng, Hydrothermally tailor-made chitosan fiber for micro-solid phase extraction of petroleum acids in crude oils, J. Chromatogr. A 1564 (2018) 42-50.

[37] W. Gan, Y. Gu, J. Han, C. Li, J. Sun, P. Liu, Chitosan-modified filter paper for nucleic acid extraction and "in situ PCR" on a thermoplastic microchip, Anal. Chem. 89 (2017) 3568-3575.

[38] J.-J. Xu, M. An, R. Yang, Z. Tan, J. Hao, J. Cao, L.-Q. Peng, W. Cao, Determination of tetracycline antibiotic residues in honey and milk by miniaturized solid phase extraction using chitosan-modified graphitized multiwalled carbon nanotubes, J. Agric. Food Chem. 64 (2016) 2647-2654.

[39] Y. Li, Z. Li, W. Wang, S. Zhong, J. Chen, A.-J. Wang, Miniaturization of selfassembled solid phase extraction based on graphene oxide/chitosan coupled with liquid chromatography for the determination of sulfonamide residues in egg and honey, J. Chromatogr. A 1447 (2016) 17-25.

[40] G.P. Mashile, A. Mpupa, P.N. Nomngongo, In-syringe micro solid-phase extraction method for the separation and preconcentration of parabens in environmental water samples, Molecules 23 (2018) 1450.

[41] H. Shu, Y. Ge, X.-Y. Xu, P.-Q. Guo, Z.-M. Luo, W. Du, C. Chang, R.-L. Liu, Q. Fu, Hybrid-type carbon microcoil-chitosan composite for selective extraction of aristolochic acid I from Aristolochiaceae medicinal plants, J. Chromatogr. A 1561 (2018) 13-19.

[42] M.E.I. Badawy, M.A.M. El-Nouby, A.E.M. Marei, Development of a solid-phase extraction (SPE) cartridge based on chitosan-metal oxide nanoparticles (ChMO NPs) for extraction of pesticides from water and determination by HPLC, Int. J. Anal. Chem. (2018) 3640691.

[43] P. Klongklaew, T. Naksena, P. Kanatharana, O. Bunkoed, A hierarchically porous composite monolith polypyrrole/octadecyl silica/graphene oxide/ chitosan cryogel sorbent for the extraction and pre-concentration of carbamate pesticides in fruit juices, Anal. Bioanal. Chem. 410 (2018) 7185-7193. 
[44] M. Asiabi, A. Mehdinia, A. Jabbari, Spider-web-like chitosan/MIL-68(Al) composite nanofibers for high-efficient solid phase extraction of $\mathrm{Pb}$ (II) and Cd(II), Microchim. Acta 184 (2017) 4495-4501.

[45] M. Asiabi, A. Mehdinia, A. Jabbari, Electrospun biocompatible Chitosan/MIL101 (Fe) composite nanofibers for solid-phase extraction of ${ }^{\Delta} 9$ tetrahydrocannabinol in whole blood samples using Box-Behnken experimental design, J. Chromatogr. A 1479 (2017) 71-80.

[46] S.S. Bozkurt, D. Erdogan, M. Antep, N. Tuzmen, M. Merdivan, Use of ionic liquid based chitosan as sorbent for preconcentration of fluoroquinolones in milk, egg, fish, bovine and chicken meat samples by solid phase extraction before HPLC determination, J. Liq. Chromatogr. Relat. Technol. 39 (2016) 21-29.

[47] C. Kong, E.K. Fodjo, D. Li, Y. Cai, D. Huang, Y. Wang, X. Shen, Chitosan-based adsorption and freeze deproteinization: improved extraction and purification of synthetic colorants from protein-rich food samples, Food Chem. 188 (2015) 240-247.

[48] N.N. Naing, S.F.Y. Li, H.K. Lee, Application of porous membrane-protected chitosan microspheres to determine benzene, toluene, ethylbenzene, xylenes and styrene in water, J. Chromatogr. A 1448 (2016) 42-48.

[49] N.N. Naing, S.F.Y. Li, H.K. Lee, Micro-solid phase extraction followed by thermal extraction coupled with gas chromatography-mass selective detector for the determination of polybrominated diphenyl ethers in water, J. Chromatogr. A 1458 (2016) 25-34.

[50] X.-M. He, X.-C. Liang, X. Chen, B.-F. Yuan, P. Zhou, L.-N. Zhang, Y.-Q. Feng, High strength and hydrophilic chitosan microspheres for the selective enrichment of N-glycopeptides, Anal. Chem. 89 (2017) 9712-9721.

[51] J.-J. Xu, Q. Li, J. Cao, E. Warner, M. An, Z. Tan, S.-L. Wang, L.-Q. Peng, X.-G. Liu, Extraction and enrichment of natural pigments from solid samples using ionic liquids and chitosan nanoparticles, J. Chromatogr. A 1463 (2016) $32-41$.

[52] N. Razavi, A.S. Yazdi, New application of chitosan-grafted polyaniline in dispersive solid-phase extraction for the separation and determination of phthalate esters in milk using high-performance liquid chromatography, J. Separ. Sci. 40 (2017) 1739-1746.

[53] W. Cao, S.-S. Hu, L.-H. Ye, J. Cao, J.-J. Xu, X.-Q. Pang, Trace-chitosan-wrapped multi-walled carbon nanotubes as a new sorbent in dispersive micro solidphase extraction to determine phenolic compounds, J. Chromatogr. A 1390 (2015) 13-21.

[54] S. Li, M. Jia, H. Guo, X. Hou, Development and application of metal organic framework/chitosan foams based on ultrasound-assisted solid-phase extraction coupling to UPLC-MS/MS for the determination of five parabens in water, Anal. Bioanal. Chem. 410 (2018) 6619-6632.

[55] W. Wu, W. Qian, H. Hao, Y. Kang, Y. Wang, Y. Deng, C. Ni, J. Huang, W. Weng, Determination of caffeoylquinic acid derivatives in Azolla imbricata by chitosan-based matrix solid-phase dispersion coupled with HPLC-PDA J. Pharm. Biomed. Anal. 163 (2019) 197-203.

[56] L.-Q. Peng, Q. Li, Y. C, M. An, R. Yang, Z. Tan, J. Hao, J. Cao, J.-J. Xu, S.-S. Hu, Determination of natural phenols in olive fruits by chitosan assisted matrix solid-phase dispersion microextraction and ultrahigh performance liquid chromatography with quadrupole time-of-flight tandem mass spectrometry, J. Cromatogr. A 1456 (2016) 68-76.

[57] H. Bagheri, A. Roostaie, M.Y. Baktash, A chitosan-polypyrrole magnetic nanocomposite as $\mu$-sorbent for isolation of naproxen, Anal. Chim. Acta 816 (2014) $1-7$

[58] Q.G. Liao, D.G. Wang, L.G. Luo, Chitosan-poly(m-phenylenediamine)@ $\mathrm{Fe}_{3} \mathrm{O}_{4}$ nanocomposite for magnetic solid-phase extraction of polychlorinated biphenyls from water samples, Anal. Bioanal. Chem. 406 (2014) 7571-7579.

[59] X. Jiang, J. Cheng, H. Zhou, F. Li, W. Wu, K. Ding, Polyaniline-coated chitosanfunctionalized magnetic nanoparticles: preparation for the extraction and analysis of endocrine-disrupting phenols in environmental water and juice samples, Talanta 141 (2015) 239-246.

[60] S. Qin, L. Su, P. Wang, Y. Gao, Rapid and selective extraction of multiple sulfonamides from aqueous samples based on $\mathrm{Fe}_{3} \mathrm{O}_{4}$-chitosan molecularly imprinted polymers, Anal. Methods 7 (2015) 8704-8713.

[61] Q.G. Liao, L.F. Hu, L.G. Luo, A chitosan-polypyrrole@ $\mathrm{Fe}_{3} \mathrm{O}_{4}$ nanocomposite for magnetic solid-phase extraction of macrolides from swine urine samples, Anal. Methods 7 (2015) 2806-2812.

[62] A. Feizbakhsh, S. Ehteshami, Polythiophene-chitosan magnetic nanocomposite as a novel sorbent for disperse magnetic solid phase extraction of triazine herbicides in aquatic media, Chromatographia 79 (2016) 1177-1185.

[63] Y. Chen, X. Ma, M. Huang, J. Peng, C. Li, Use of a new magnetic ion-imprinted nanocomposite adsorbent for selective and rapid preconcentration and determination of trace nickel by flame atomic absorption spectrometry, Anal. Methods 8 (2016) 824-829.

[64] A. Feizbakhsh, S. Ehteshami, Modified magnetic nanoparticles as a novel sorbent for dispersive magnetic solid-phase extraction of triazine herbicides in aqueous media, J. AOAC Int. 100 (2017) 198-205.

[65] E.Ö. Er, E. Maltepe, S. Bakirdere, A novel analytical method for the determination of cadmium in sorrel and rocket plants at ultratrace levels: magnetic chitosan hydrogels based solid phase microextraction-slotted quartz tube-flame atomic absorption spectrophotometry, Microchem. J. 143 (2018) 393-399.

[66] N. Razavi, Z. Es'haghi, Employ of magnetic polyaniline coated chitosan nanocomposite for extraction and determination of phthalate esters in diapers and wipes using gas chromatography, Microchem. J. 142 (2018) 359-366.

[67] B. Fahimirad, Y. Rangraz, A. Elhampour, F. Nemati, Diphenyl diselenide grafted onto a $\mathrm{Fe}_{3} \mathrm{O}_{4}$-chitosan composite as a new nanosorbent for separation of metal ions by effervescent salt-assisted dispersive magnetic micro solid-phase extraction, Microchim. Acta 185 (2018) 560.

[68] B.G. Maciel, R.J. da Silva, A.E. Chávez-Guajardo, J.C. Medina-Llamas, J.J. Alcaaraz-Espinoza, C.P. de Melo, Magnetic extraction and purification of DNA from whole human blood using a $\gamma$ - $\mathrm{Fe}_{2} \mathrm{O}_{3} @$ Chitosan@Polyaniline hybrid nanocomposite, Carbohydr. Polym. 197 (2018) 100-108.

[69] W. Ma, Y. Dai, K.H. Row, Molecular imprinted polymers based on magnetic chitosan with different deep eutectic solvent monomers for the selective separation of catechins in black tea, Electrophoresis 39 (2018) 2039-2046.

[70] R. Wang, Y. Cui, F. Hu, W. Liu, Q. Du, Y. Zhang, J. Zha, T. Huang, M. Fizir, H. He Selective recognition and enrichment of carbamazepine in biological samples by magnetic imprinted polymer based on reversible additionfragmentation chain transfer polymerization, J. Chromatogr. A 1591 (2019) $62-70$.

[71] S.R. Bandforuzi, M.R. Hadjmohammadi, Modified magnetic chitosan nanoparticles based on mixed hemimicelle of sodium dodecyl sulfate for enhanced removal and trace determination of three organophosphorus pesticides from natural waters, Anal. Chim. Acta 1078 (2019) 90-100.

[72] Q. Tang, X. Wang, F. Yu, X. Qiao, Z. Xu, Simultaneous determination of ten organophosphate pesticide residues in fruits by gas chromatography coupled with magnetic separation, J. Separ. Sci. 37 (2014) 820-827.

[73] T. Liu, J. Xie, J. Zhao, G. Song, Y. Hu, Magnetic chitosan nanocomposite used as cleanup material to detect chloramphenicol in milk by GC-MS, Food Anal. Methods 7 (2014) 814-819.

[74] C. Cui, M. He, B. Chen, B. Hu, Chitosan modified magnetic nanoparticles based solid phase extraction combined with ICP-OES for the speciation of $\mathrm{Cr}$ (III) and $\mathrm{Cr}(\mathrm{VI})$, Anal. Methods 6 (2014) 8577-8583.

[75] E. Ziaei, A. Mehdinia, A. Jabbari, A novel hierarchical nanobiocomposite of graphene oxide-magnetic chitosan grafted with mercapto as a solid phase extraction sorbent for the determination of mercury ions in environmental water samples, Anal. Chim. Acta 850 (2014) 49-56.

[76] L. Zhang, Z. Xiong, L. Zhang, B. Yu, W. Zhang, Magnetic nanoparticles coated with dithizone-modified chitosan for use in solid-phase extraction of copper(II), Anal. Methods 7 (2015) 2050-2054.

[77] X. Ding, Y. Wang, Y. Wang, Q. Pan, J. Chen, Y. Huang, K. Xu, Preparation of magnetic chitosan and graphene oxide-functional guanidinium ionic liquid composite for the solid-phase extraction of protein, Anal. Chim. Acta 861 (2015) 36-46.

[78] N.N. Naing, S.F.Y. Li, H.K. Lee, Magnetic micro-solid-phase-extraction of polycyclic aromatic hydrocarbons in water, J. Chromatogr. A 1440 (2016) 23-30.

[79] S.J. Sadeghi, S. Seidi, J.B. Ghasemi, Graphene oxide-alizarin yellow $\mathrm{R}$-magnetic chitosan nanocomposite: a selective and efficient sorbent for sub-trace determination of aluminum in water samples, Anal. Methods 9 (2017) 222-231.

[80] A. Barati, E. Kazemi, S. Dadfarnia, A.M.H. Shabani, Synthesis/characterization of molecular imprinted polymer based on magnetic chitosan/graphene oxide for selective separation/preconcentration of fluoxetine from environmental and biological samples, J. Ind. Eng. Chem. 46 (2017) 212-221.

[81] E. Kazemi, S. Dadfarnia, A.M.H. Shabani, M. Ranjbar, Synthesis, characterization, and application of a $\mathrm{Zn}$ (II)-imprinted polymer grafted on graphene oxide/magnetic chitosan nanocomposite for selective extraction of zinc ions from different food samples, Food Chem. 237 (2017) 921-928.

[82] K. Xu, Y. Wang, H. Zhang, Q. Yang, X. Wei, P. Xu, Y. Zhour, Solid-phase extraction of DNA by using a composite prepared from multiwalled carbon nanotubes, chitosan, $\mathrm{Fe}_{3} \mathrm{O}_{4}$ and a poly(ethylene glycol)-based deep eutectic solvent, Microchim. Acta 184 (2017) 4133-4140.

[83] J. Shah, M.R. Jan, Tasmia, Magnetic chitosan graphene oxide composite for solid phase extraction of phenylurea herbicides, Carbohydr. Polym. 199 (2018) 461-472.

[84] J. Xie, H. Jiang J. Shen, T. Peng, J. Wang, K. Yao, S. Sun, B. Shao, J. Tang, Design of multifunctional nanostructure for ultrafast extraction and purification of aflatoxins in foodstuffs, Anal. Chem. 89 (2017) 10556-10564.

[85] A.P. Tiwari, R.K. Satvekar, S.S. Rohiwal, V.A. Karande, A.V. Raut, P.G. Patil, P.B. Shete, S.J. Ghosh, S.H. Pawar, Magneto-separation of genomic deoxyribose nucleic acid using pH responsive $\mathrm{Fe}_{3} \mathrm{O}_{4} @$ silica@chitosan nanoparticles in biological samples, RSC Adv. 6 (2015) 8463-8470.

[86] H. Sereshti, S. Samadi, S. Asgari, M. Karimi, Preparation and application of magnetic graphene oxide coated with a modified chitosan $\mathrm{pH}$-sensitive hydrogel: an efficient biocompatible adsorbent for catechin, RSC Adv. 5 (2015) 9396-9404.

[87] M.K. Moazen, H.A. Panahi, Magnetic iron oxide nanoparticles grafted $\mathrm{N}$-isopropylacrylamide/chitosan copolymer for the extraction and determination of letrozole in human biological samples, J. Separ. Sci. 40 (2017) 1125-1132.

[88] M. Hashemi, Z. Nazari, N. Noshirvani, Synthesis of chitosan based magnetic molecularly imprinted polymers for selective separation and spectrophotometric determination of histamine in tuna fish, Carbohydr. Polym. 177 (2017) 306-314

[89] F. Makkliang, P. Kanatharana, P. Thavarungkul, C. Thammakhet-Buranachai, A polypyrrole-chitosan cryogel stir-bead micro-solid phase extractor for the determination of phthalate esters in contact lenses storage solutions and in 
artificial saliva in contact with baby teethers, Anal. Chim. Acta 985 (2017) 69-78.

[90] D. Xiao, C. Zhang, D. Yuan, J. He, J. Wu, K. Zhang, R. Lin, H. Hee, Magnetic solid-phase extraction based on $\mathrm{Fe}_{3} \mathrm{O}_{4}$ nanoparticle retrieval of chitosan for the determination of flavonoids in biological samples coupled with high performance liquid chromatography, RSC Adv. 4 (2014) 64843-64854.

[91] S. Kheirandish, M. Ghaedi, K. Dashtian, F. Pourebrahim, Design of a new technique based on combination of ultrasound waves via magnetite solid phase and cloud point microextraction for determination of $\mathrm{Cr}$ (III) ions, Ultrason. Sonochem. 39 (2017) 798-809.

[92] A. Ostovan, M. Ghaedi, M. Arabi, Fabrication of water-compatible superparamagnetic molecularly imprinted biopolymer for clean separation of baclofen from bio-fluid samples: a mild and green approach, Talanta 179 (2018) 760-768.

[93] D. Ge, H.K. Lee, Polypropylene membrane coated with carbon nanotubes functionalized with chitosan: application in the microextraction of polychlorinated biphenyls and polybrominated diphenyl ethers from environmental water samples, J. Chromatogr. A 1408 (2015) 56-62.

[94] W.N.W. Ibrahim, M.M. Sanagi, N.S.M. Hanapi, S. Kamaruzaman, N. Yahaya, W.A.W. Ibrahim, Solid-phase microextraction based on an agarose-chitosanmultiwalled carbon nanotube composite film combined with HPLC-UV for the determination of nonsteroidal anti-inflammatory drugs in aqueous samples, J. Separ. Sci. 41 (2018) 2942-2951.

[95] L. Djerahov, P. Vasileva, I. Karadjova, R.M. Kurakalva, K.K. Aradhi, Chitosan film loaded with silver nanoparticles - sorbent for solid phase extraction of $\mathrm{Al}(\mathrm{III}), \mathrm{Cd}(\mathrm{II}), \mathrm{Cu}(\mathrm{II}), \mathrm{Co}(\mathrm{II}), \mathrm{Fe}(\mathrm{III}), \mathrm{Ni}(\mathrm{II}), \mathrm{Pb}(\mathrm{II})$ and $\mathrm{Zn}(\mathrm{II})$, Carbohydr. Polym. 147 (2016) 45-52.

[96] L. Djerahov, P. Vasileva, I. Karadjova, Self-standing chitosan film loaded with silver nanoparticles as a tool for selective determination of $\mathrm{Cr}(\mathrm{VI})$ by ICP-MS, Microchem. J. 129 (2016) 23-28.

[97] N.T. Ng, M.M. Sanagi, W.N.W. Ibrahim, W.A.W. Ibrahim, Agarose-chitosan$\mathrm{C}_{18}$ film micro-solid phase extraction combined with high performance liquid chromatography for the determination of phenanthrene and pyrene in chrysanthemum tea samples, Food Chem. 222 (2017) 28-34.

[98] M. Saraji, M. Tarami, N. Mehrafza, Preparation of a nano-biocomposite film based on halloysite-chitosan as the sorbent for thin film microextraction, Microchem. J. 150 (2019) 104171.

[99] R. Alizadeh, Chlorophenol's ultra-trace analysis in environmental samples by chitosan-zinc oxide nanorod composite as a novel coating for solid phase micro-extraction combined with high performance liquid chromatography, Talanta 146 (2016) 831-838.

[100] S. Wu, C. Cai, J. Cheng, M. Cheng, H. Zhou, J. Deng, Polydopamine/dialdehyde starch/chitosan composite coating for in-tube solid-phase microextraction and in-situ derivation to analysis of two liver cancer biomarkers in human blood, Anal. Chim. Acta 935 (2016) 113-120.

[101] C. Ruiz-Palomero, M.L. Soriano, M. Valcárcel, $\beta$-Cyclodextrin decorated nanocellulose: a smart approach towards the selective fluorimetric determination of danofloxacin in milk samples, Analyst 140 (2015) 3431-3438.

[102] I. López-García, J.J. Marín-Hernández, M. Hernández-Córdoba, Microcrystalline cellulose for the dispersive solid phase microextraction and sensitive determination of chromium in water using electrothermal atomic absorption spectrometry, J. Anal. At. Spectrom. 33 (2018) 1529-1535.

[103] D. Zhang, L. Zhang, T. Liu, A magnetic cellulose-based carbon fiber hybrid as a dispersive solid-phase extraction material for the simultaneous detection of six bisphenol analogs from environmental samples, Analyst 143 (2018) 3100-3106.

[104] C. Ye, Y. Wu, Z. Wang, Modification of cellulose paper with polydopamine as a thin film microextraction phase for detection of nitrophenols in oil samples, RSC Adv. 6 (2016) 9066-9071.

[105] V. Samanidou, L.-D. Galanopoulos, A. Kabir, K.G. Furton, Fast extraction of amphenicols residues from raw milk using novel fabric phase sorptive extraction followed by high-performance liquid chromatography-diode array detection, Anal. Chim. Acta 855 (2015) 41-50.

[106] R. Kaur, R. Kaur, S. Rani, A.K. Malik, A. Kabir, K.G. Furton, Application of fabric phase sorptive extraction with gas chromatography and mass spectrometry for the determination of organophosphorus pesticides in selected vegetable samples, J. Separ. Sci. 42 (2019) 862-870.

[107] J. Wang, Q. Yang, L. Zhang, M. Liu, N. Hu, W. Zhang, W. Zhu, R. Wang, Y. Suo, J. Wang, A hybrid monolithic column based on layered double hydroxidealginate hydrogel for selective solid phase extraction of lead ions in food and water samples, Food Chem. 257 (2018) 155-162.

[108] A.S. Abboud, M.M. Sanagi, W.A.W. Ibrahim, A.S.A. Keyon, H.Y. Aboul-Enein, Calcium alginate-caged multiwalled carbon nanotubes dispersive microsolid phase extraction combined with gas chromatography-flame ionization detection for the determination of polycyclic aromatic hydrocarbons in water samples, J. Chromatogr. Sci. 56 (2018) 177-186.

[109] O. Bunkoed, P. Nurerk, R. Wannapob, P. Kanatharana, Polypyrrole-coated alginate/magnetite nanoparticles composite sorbent for the extraction of endocrine-disrupting compounds, J. Separ. Sci. 39 (2016) 3602-3609.

[110] M.M. Sanagi, M.N.M.M. Jais, S. Kamaruzaman, W.A.W. Ibrahim, U. Baig, Multi-walled carbon nanotubes-agarose gel micro-solid phase extraction for the determination of triazine herbicides in water samples, Anal. Methods 7 (2015) 2862-2868.

[111] N. Poursheikhi, P. Hashemi, M. Safdarian, F.N. Serenjeh, F. Hesami, Agarose based magnetic solid-phase extraction-magnetic field agitation for determination of trace amounts of molybdenum in beans, J. Braz. Chem. Soc. 27 (2016) 670-676.

[112] M.N.H. Rozaini, N. Semail, B. Saad, S. Kamaruzaman, W.N. Abdullah, N.A. Rahim, M. Miskam, S.H. Loh, N. Yahaya, Molecularly imprinted silica gel incorporated with agarose polymer matrix as mixed matrix membrane for separation and preconcentration of sulfonamide antibiotics in water samples, Talanta 199 (2019) 522-531.

[113] M. Zhang, G. Ma, L. Zhang, H. Chen, L. Zhu, C. Wang, X. Liu, Chitosan-reduced graphene oxide composites with 3D structures as effective reverse dispersed solid phase extraction adsorbents for pesticides analysis, Analyst 144 (2019) 5164.

[114] L.C. Cabrera, S.S. Caldas, O.D. Prestes, E. Prime, R. Zanella, Evaluation of alternative sorbents for dispersive solid-phase extraction clean-up in the QuEChERS method for the determination of pesticide residues in rice by liquid chromatography with tandem mass spectrometry, J. Separ. Sci. 39 (2016) 1945-1954.

[115] D. Klemm, B. Heublein, H.-P. Fink, A. Bohn, Cellulose: fascinating biopolymer and sustainable raw material, Angew. Chem. Int. Ed. 44 (2005) 3358-3393.

[116] J. Shojaeiarani, D. Bajwa, A. Shirzadifar, A review on cellulose nanocrystals as promising biocompounds for the synthesis of nanocomposite hydrogels, Carbohydr. Polym. 216 (2019) 247-259.

[117] H. Kargarzadeh, M. Mariaano, D. Gopakumar, I. Ahmad, S. Thomas, A. Dufresne, J. Huang, N. Lin, Advances in cellulose nanomaterials, Cellulose 25 (2018) 2151-2189.

[118] L.A.C. Minho, G.C. Brandão, D.A. Santana, O.S. Santos, H.M.C. Andrade, W.N.L. dos Santos, Cellulose-coated $\mathrm{CoFe}_{2} \mathrm{O}_{4}$ nanoparticles as an adsorbent for extraction and preconcentration of bioactive compounds in vinegars, Microchem. J. 147 (2019) 102-111.

[119] T.G. Aqda, S. Behkami, H. Bagheri, Porous eco-friendly fibers for on-line micro solid-phase extraction of nonsteroidal anti-inflammatory drugs from urine and plasma samples, J. Chromatogr. A 1574 (2018) 18-26.

[120] J. Cao, L.-Q. Peng, L.-J. Du, Q.-D. Zhang, J.-J. Xu, Ultrasound-assisted ionic liquid-based micellar extraction combined with microcrystalline cellulose as sorbent in dispersive microextraction for the determination of phenolic compounds in propolis, Anal. Chim. Acta 963 (2017) 24-32.

[121] C. Ruiz-Palomero, M.L. Soriano, M. Valcárcel, Sulfonated nanocellulose for the efficient dispersive micro solid-phase extraction and determination of silver nanoparticles in food products, J. Chromatogr. A 1428 (2016) 352-358.

[122] J. Cao, L.-Q. Peng, J.-J. Xu, Microcrystalline cellulose based matrix solid phase dispersionmicroextration for isomeric triterpenoid acids in loquat leaves by ultrahigh-performance liquid chromatography and quadrupole time-offlight mass spectrometry, J. Chromatogr. A 1472 (2016) 16-26.

[123] F. Abujaber, M. Zougagh, S. Jodeh, Á. Ríos, F.J.G. Bernardo, R.C.R. MartínDoimeadios, Magnetic cellulose nanoparticles coated with ionic liquid as a new material for the simple and fast monitoring of emerging pollutants in waters by magnetic solid phase extraction, Microchem. J. 137 (2018) 490-495.

[124] Z. Li, S. Lu, J. Jin, T. Wang, Preparation of a new cellulose magnetic molecularly imprinted polymer micro-spheres to extract and analyze the indole-3acetic acid in plant tissues, J. Chromatogr. B 1092 (2018) 343-349.

[125] X. Meng, Q. Liu, Y. Ding, Paper-based solid-phase microextraction for analysis of 8-hydroxy-2'-deoxyguanosine in urine sample by CE-LIF, Electrophoresis 38 (2017) 494-500.

[126] S.H. Loh, E.L. Aw, S.W. Lee, Extraction of bisphenol A in environmental water and soft drink by $\mathrm{C}_{18}$-impregnated cellulose triacetate composite film, Malaysian J. Anal. Sci. 22 (2018) 383-390.

[127] L. Wan, H. Zhu, Y. Guan, G. Huang, Nanocoating cellulose paper based microextraction combined with nanospray mass spectrometry for rapid and facile quantitation of ribonucleosides in human urine, Talanta 169 (2017) 209-215.

[128] Z. Hashemian, T. Khayamian, M. Saraji, Anticodeine aptamer immobilized on a Whatman cellulose paper for thin-film microextraction of codeine from urine followed by electrospray ionization ion mobility spectrometry, Anal. Bioanal. Chem. 407 (2015) 1615-1623.

[129] N.H. Mukhtar, H.H. See, Carbonaceous nanomaterials immobilised mixed matrix membrane microextraction for the determination of polycyclic aromatic hydrocarbons in sewage pond water samples, Anal. Chim. Acta 931 (2016) 57-63.

[130] A. Kabir, K.G. Furton, Fabric Phase Sorptive Extractor (FPSE), U.S. Patent and Trademark Office 14,216,121, 17 March 2014.

[131] V. Samanidou, I. Kaltzi, A. Kabir, K.G. Furton, Simplifying sample preparation using fabric phase sorptive extraction technique for the determination of benzodiazepines in blood serum by high-performance liquid chromatography, Biomed. Chromatogr. 30 (2016) 829-836.

[132] M. Roldán-Pijuán, R. Lucena, S. Cárdenas, M. Valcárcel, A. Kabir, K.G. Furton, Stir fabric phase sorptive extraction for the determination of triazineherbicides in environmental waters by liquid chromatography, J. Chromatogr. A 1376 (2015) 35-45.

[133] E. Karageorgou, N. Manousi, V. Samanidou, A. Kabir, K.G. Furton, Fabric phase sorptive extraction for the fast isolation of sulfonamides residues from raw milk followed by high performance liquid chromatography with ultraviolet detection, Food Chem. 196 (2016) 428-436.

[134] R. Kaur, R. Kaur, S. Rani, A.K. Malik, A. Kabir, K.G. Furton, V.F. Samanidou, Rapid monitoring of organochlorine pesticide residues in various fruit juices and water samples using fabric phase sorptive extraction and gas chromatography-mass spectrometry, Molecules 24 (2019) 1013. 
[135] L. Pérez-Mayán, I. Rodríguez, M. Ramil, A. Kabir, K.G. Furton, R. Cela, Fabric phase sorptive extraction followed by ultra-performance liquid chromatography-tandem mass spectrometry for the determination of fungicides and insecticides in wine, J. Chromatogr. A 1584 (2019) 13-23.

[136] R.G. Alonso, L. Ciofi, Z.S. Ferrera, J.J.S. Rodríguez, M. Bubba, A. Kabir, K.G. Furton, Determination of androgens and progestogens in environmental and biological samples using fabric phase sorptive extraction coupled to ultra-high performance liquid chromatography tandem mass pectrometry, J. Chromatogr. A 1437 (2016) 116-126.

[137] F. Abujaber, F.J.G. Bernardo, R.C.R. Martín-Doimeadios, Magnetic cellulose nanoparticles as sorbents for stir bar-sorptive dispersive microextraction of polychlorinated biphenyls in juice samples, Talanta 201 (2019) 266-270.

[138] D.-E. Georgiadis, A. Tsalbouris, A. Kabir, K.G. Furton, V. Samanidou, Novel capsule phase microextraction in combination with high performance liquid chromatography with diode array detection for rapid monitoring of sulfonamide drugs in milk, J. Separ. Sci. 42 (2019) 1440-1450.

[139] K.Y. Lee, D.J. Mooney, Alginate: properties and biomedical applications, Prog. Polym. Sci. 37 (2012) 106-126.

[140] C. Alvarez-Loreno, B. Blanco-Fernandez, A.M. Puga, A. Concheiro, Crosslinked ionic polysaccharides for stimuli-sensitive drug delivery, Adv. Drug Deliv. Rev. 65 (2013) 1148-1171.

[141] M.S. Tabish, N.S.M. Hanapi, W.N.W. Ibrahim, N. Saim, N. Yahaya, Alginategraphene oxide biocomposite sorbent for rapid and selective extraction of non-steroidal anti-inflammatory drugs using micro-solid phase extraction, Indones. J. Chem. 19 (2019) 684-695.

[142] M. Zare, Z. Ramezani, N. Rahbar, Development of zirconia nanoparticlesdecorated calcium alginate hydrogel fibers for extraction of organophosphorous pesticides from water and juice samples: facile synthesis and application with elimination of matrix effects, J. Chromatogr. A 1473 (2016) 28-37.

[143] O. Bunkoed, P. Kanatharana, Extraction of polycyclic aromatic hydrocarbons with a magnetic sorbent composed of alginate, magnetite nanoparticles and multiwalled carbon nanotubes, Microchim. Acta 182 (2015) 1519-1526.

[144] P. Nurerk, P. Kanatharana, O. Bunkoed, Polyaniline-coated magnetite nanoparticles incorporated in alginate beads for the extraction and enrichment of polycyclic aromatic hydrocarbons in water samples, Int. J. Environ. Anal. Chem. 97 (2017) 145-158.

[145] S.C. Tan, H.K. Lee, A hydrogel composite prepared from alginate, an aminofunctionalized metal-organic framework of type MIL-101(Cr), and magnetite nanoparticles for magnetic solid-phase extraction and UHPLC-MS/MS analysis of polar chlorophenoxy acid herbicides, Microchim. Acta 186 (2019) 545.

[146] N.D.B. Castilhos, N.M.F.M. Sampaio, B.C. da Silvaa, I.C. Riegel-Vidotti, M.T. Grassi, B.J.G. Silva, Physical-chemical characteristics and potential use of a novel alginate/zein hydrogel as the sorption phase for polar organic compounds, Carbohydr. Polym. 174 (2017) 507-516.

[147] S. Graham, P.F. Marina, A. Blencowe, Thermoresponsive polysaccharides and their thermoreversible physical hydrogel networks, Carbohydr. Polym. 207 (2019) 143-159.

[148] P. Zarrintaj, S. Manouchehri, Z. Ahmadi, M.R. Saeb, A.M. Urbanska, D.L. Kaplan, M. Mozafari, Agarose-based biomaterials for tissue engineering, Carbohydr. Polym. 187 (2018) 66-84.

[149] C. Huang, Z. Chen, A. Gjelstad, S. Pedersen-Bjergaard, X. Shen, Electromembrane extraction, Trac. Trends Anal. Chem. 95 (2017) 47-56.

[150] N. Pourmand, M.M. Sanagi, A.A. Naim, W.A.W. Ibrahim, U. Baig, Dispersive micro-solid phase extraction method using newly prepared poly(methyl methacrylate) grafted agarose combined with ICP-MS for the simultaneous determination of $\mathrm{Cd}, \mathrm{Ni}, \mathrm{Cu}$ and $\mathrm{Zn}$ in vegetable and natural water samples, Anal. Methods 7 (2015) 3215-3223.
[151] F.N. Serenjeh, P. Hashemi, H. Naeimi, E. Zakerzadeh, A.R. Ghiasvand, Spherical agarose-coated magnetic nanoparticles functionalized with a new salen for magnetic solid-phase extraction of uranyl ion, Microchim. Acta 183 (2016) 2449-2455.

[152] H. Lu, A. Lu, H. Fu, B. Li, M. Yang, J. Wang, Y. Luan, Affinity capture of aflatoxin $\mathrm{B}_{1}$ and $\mathrm{B}_{2}$ by aptamer-functionalized magnetic agarose microspheres prior to their determination by HPLC, Microchim. Acta 185 (2018) 326.

[153] S.H. Loh, P.E. Neoh, C.T. Tai, S. Kamaruzaman, Simple $\mu$-solid phase extraction using $\mathrm{C}_{18}$ film for the extraction of polycyclic aromatic hydrocarbons in coffee beverage, Malaysian J. Anal. Sci. 22 (2018) 1-7.

\section{List of abbreviations}

M-dSPE: Dispersive miniaturized solid-phase extraction
M-SPE: Miniaturized solid-phase extraction
AAS: Atomic absorption spectroscopy
BPS: Bisphenols
CNTS: Carbon nanotubes
COFs: Covalent organic frameworks
DES: Deep eutectic solvent
DAD: Diode-array detection
DI-SPME: Direct immersion solid phase microextraction
EME: Electromembrane extraction
FAAS: Flame atomic absorption spectroscopy
FD: Fluorescence detection
FID: Flame ionization detection
FPSE: Fabric-phase sorptive extraction
GAC: Green analytical chemistry
GC: Gas chromatography
GFAAS: Graphite furnace atomic absorption spectroscopy
GO: graphene oxide
ICP: Inductively coupled plasma
IL: Ionic liquid
LC: Liquid-chromatography
LOD: Limit of detection
LOQ: Limit of quantification
m- $\boldsymbol{\mu}$-dSPE: Magnetic-assisted dispersive miniaturized solid-phase extraction
MIPs: Molecularly imprinted polymers
MNPS: Magnetic nanoparticles
MOFs: Metal-organic frameworks
MS: Mass spectrometry
MS/MS: Tandem mass spectrometry
MSPD: Matrix solid-phase dispersion
MWCNTs: Multi-walled carbon nanotubes
OES: Optical emission spectrometry
PAEs: Phthalate acid esters
PAHs: Polycyclic aromatic hydrocarbons
PBBs: Polybrominated biphenyls
PCBs: Polychlorinated biphenyls
PEG: Polyethylene glycol
SBSE: Stir bar sorptive extraction
SPE: Solid-phase extraction
SPME: Solid-phase microextraction
STE: Sorptive tape extraction
TFME: Thin-film microextraction

